\title{
Three Positive Periodic Solutions to Nonlinear Neutral Functional Differential Equations with Parameters on Variable Time Scales
}

\author{
Yongkun Li and Chao Wang \\ Department of Mathematics, Yunnan University, Yunnan, Kunming 650091, China \\ Correspondence should be addressed to Yongkun Li, yklie@ynu.edu.cn
}

Received 20 October 2011; Revised 19 December 2011; Accepted 25 December 2011

Academic Editor: Laurent Gosse

Copyright (C) 2012 Y. Li and C. Wang. This is an open access article distributed under the Creative Commons Attribution License, which permits unrestricted use, distribution, and reproduction in any medium, provided the original work is properly cited.

\begin{abstract}
Using two successive reductions: B-equivalence of the system on a variable time scale to a system on a time scale and a reduction to an impulsive differential equation and by Leggett-Williams fixed point theorem, we investigate the existence of three positive periodic solutions to the nonlinear neutral functional differential equation on variable time scales with a transition condition between two consecutive parts of the scale $(d / d t)(x(t)+c(t) x(t-\alpha))=a(t) g(x(t)) x(t)-\sum_{j=1}^{n} \lambda_{j} f_{j}(t, x(t-$ $\left.\left.v_{j}(t)\right)\right),(t, x) \in \mathbb{T}_{0}(x),\left.\Delta t\right|_{(t, x) \in \mathcal{S}_{2 i}}=\Pi_{i}^{1}(t, x)-t,\left.\Delta x\right|_{(t, x) \in \mathcal{S}_{2 i}}=\Pi_{i}^{2}(t, x)-x$, where $\Pi_{i}^{1}(t, x)=t_{2 i+1}+$ $\tau_{2 i+1}\left(\Pi_{i}^{2}(t, x)\right)$ and $\Pi_{i}^{2}(t, x)=B_{i} x+J_{i}(x)+x, i=1,2, \ldots \lambda_{j}(j=1,2, \ldots, n)$ are parameters, $\mathbb{T}_{0}(x)$ is a variable time scale with $(\omega, p)$-property, $c(t), a(t), v_{j}(t)$, and $f_{j}(t, x)(j=1,2, \ldots, n)$ are $\omega$ periodic functions of $t, B_{i+p}=B_{i}, J_{i+p}(x)=J_{i}(x)$ uniformly with respect to $i \in \mathbb{Z}$.
\end{abstract}

\section{Introduction}

In the last several decades, the theory of dynamic equations on time scales (DETS) has been developed very intensively. For the full description of the equations we refer to the nicely written books $[1,2]$ and papers $[3,4]$. The equations have a very special transition condition for adjoint elements of time scales. To enlarge the field of applications of the DETS, Akhmet and Turan proposed to generalize the transition operator [5], correspondingly to investigate differential equations on variable time scales with transition condition (DETC). In [6], Akhmet and Turan proposed some basic theory of dynamic equations on variable time scales; the method of investigation is by means of two successive reductions: $B$-equivalence of the system [7-9] on a variable time scale to a system on a time scale and a reduction to an impulsive differential equation $[5,7]$. Consequently, these results are very effective to develop methods of investigation of mechanical models with impacts. 
Also, neutral differential equations arise in many areas of applied mathematics, and for this reason these equations have received much attention in the last few decades; they are not only an extension of functional differential equations but also provide good models in many fields including biology, mechanics, and economics. In particular, qualitative analysis such as periodicity and stability of solutions of neutral functional differential equations has been studied extensively by many authors. We refer to [10-19] for some recent work on the subject of periodicity and stability of neutral equations. In [20], the authors discussed a class of neutral functional differential equations with impulses and parameters on nonvariable time scales

$$
\begin{gathered}
\left(x(t)+c(t) x\left(t-r_{1}\right)\right)^{\Delta}=a(t) g(x(t)) x(t)-\sum_{i=1}^{n} \lambda_{i} f_{i}\left(t, x\left(t-\tau_{i}(t)\right)\right), \\
t \neq t_{j}, t \in \mathbb{T}, j=1,2, \ldots, q, \\
x\left(t_{j}^{-}\right)-x\left(t_{j}^{+}\right)=I_{j}\left(x\left(t_{j}\right)\right), \quad t=t_{j}, j=1,2, \ldots, q,
\end{gathered}
$$

where $\lambda_{i}, i=1,2, \ldots, n$ are parameters, $\mathbb{T}$ is an $\omega$-periodic nonvariable time scale, $a \in$ $C\left(\mathbb{T}, \mathbb{R}^{+}\right), c \in C(\mathbb{T},[0,1))$ and both of them are $\omega$-periodic functions, $\tau_{i} \in C(\mathbb{T}, \mathbb{R}), \quad i=$ $1,2, \ldots, n$ are $\omega$-periodic functions, $f_{i} \in C\left(\mathbb{T} \times \mathbb{R}^{+}, \mathbb{R}^{+}\right), i=1,2, \ldots, n$ are nondecreasing with respect to their second arguments and $\omega$-periodic with respect to their first arguments, respectively; $g \in C\left(\mathbb{R}, \mathbb{R}^{+}\right)$and there exist two positive constants $l, L$ such that $0<l \leq g(x) \leq$ $L<\infty$ for all $x>0, I_{j} \in C\left(\mathbb{R}, \mathbb{R}^{+}\right)(j=1,2, \ldots, q)$ and is bounded, $r_{1}$ is a constant.

To the best of authors' knowledge, there has been no paper published on the existence of solutions to neutral functional differential equations on variable time scales. Our main purpose of this paper is by using theory of dynamic equations on variable time scales to investigate the existence of three positive periodic solutions to the nonlinear neutral functional differential equations on variable time scales with a transition condition between two consecutive parts of the scale

$$
\begin{aligned}
\frac{\mathrm{d}}{\mathrm{d} t}(x(t)+c(t) x(t-\alpha))=a(t) g(x(t)) x(t) & -\sum_{j=1}^{n} \lambda_{j} f_{j}\left(t, x\left(t-v_{j}(t)\right)\right), \quad(t, x) \in \mathbb{T}_{0}(x), \\
\left.\Delta t\right|_{(t, x) \in \mathcal{S}_{2 i}} & =\Pi_{i}^{1}(t, x)-t, \\
\left.\Delta x\right|_{(t, x) \in \mathcal{S}_{2 i}} & =\Pi_{i}^{2}(t, x)-x,
\end{aligned}
$$

where $\Pi_{i}^{1}(t, x)=t_{2 i+1}+\tau_{2 i+1}\left(\Pi_{i}^{2}(t, x)\right)$ and $\Pi_{i}^{2}(t, x)=B_{i} x+J_{i}(x)+x, i=1,2, \ldots \lambda_{j}(j=$ $1,2, \ldots, n)$ are parameters, $\mathbb{T}_{0}(x)$ is a variable time scale with $(\omega, p)$-property, $c(t), a(t), v_{j}(t)$, and $f_{j}(t, x)(j=1,2, \ldots, n)$ are $\omega$-periodic functions of $t, B_{i+p}=B_{i}, J_{i+p}(x)=J_{i}(x)$ uniformly with respect to $i \in \mathbb{Z}$.

For convenience, we introduce the notation

$$
\bar{a}=\max _{t \in[0, \omega]} a(t), \quad \underline{a}=\min _{t \in[0, \omega]} a(t), \quad \underline{c}=\min _{t \in[0, \omega]} c(t), \quad \bar{c}=\max _{t \in[0, \omega]} c(t), \quad r_{0}=\exp \left\{\int_{\omega}^{0} a(s) \mathrm{d} s\right\} .
$$

Throughout this paper, we assume the following. 
$\left(\mathrm{H}_{1}\right) \mathbb{T}_{0}(x)$ is a variable time scale with $(\omega, p)$-property, $c \in C(\mathbb{R},[0,1))$, a $\in$ $C\left(\mathbb{R}, \mathbb{R}^{+}\right), v_{j} \in C(\mathbb{R}, \mathbb{R})$, and $f_{j}(t, x) \in C\left(\mathbb{T}_{0}(x), \mathbb{R}^{+}\right),(j=1,2, \ldots, n)$ are $\omega$-periodic functions of $t, J_{i} \in C(\mathbb{R}, \mathbb{R}), B_{i+p}=B_{i}, J_{i+p}(x)=J_{i}(x)$ uniformly with respect to $i \in \mathbb{Z}, J_{i}(0)=0, B_{i} \geq 0$ for each $i \in \mathbb{Z}$.

$\left(\mathrm{H}_{2}\right) f_{j}(t, x) \in C\left(\mathbb{T}_{0}(x), \mathbb{R}^{+}\right)$is nondecreasing with respect to $x$ and $f_{j}(t, 0)=0$ for each $j \in\{1,2, \ldots, n\}$.

$\left(\mathrm{H}_{3}\right) g \in C\left(\mathbb{R}, \mathbb{R}^{+}\right)$and there exist two positive constants $l, L$ such that $0<l \leq g(x) \leq L<$ $\infty$ for all $x>0$.

$\left(\mathrm{H}_{4}\right)$ There exists a number $\delta>0$ such that $(\underline{c}+\bar{c}) / 2 \leq \delta<1,0 \leq\left((2 \delta-\underline{c})\left(1-\underline{c}^{2}\right)\right) /(1-$ $\left.(2 \delta-\underline{c})^{2}\right)<r_{0}^{L}\left(1-r_{0}^{l}\right) /\left(1-r_{0}^{L}\right)$.

\section{Preliminaries}

Let $E$ be a real Banach space and $P$ be a cone in $E$. A map $\rho$ is said to be a nonnegative continuous concave functional on $P$ if $\rho: P \rightarrow[0, \infty)$ is continuous and

$$
\rho(t x+(1-t) y) \geq t \rho(x)+(1-t) \rho(y) \quad \forall x, y \in P \text { and } t \in[0,1]
$$

For numbers $\beta_{1}, \beta_{4}$ such that $0<\beta_{1}<\beta_{4}$ and $\rho$ is a nonnegative continuous concave function on $P$, we define the following sets: $P_{\beta_{1}}=\left\{x \in P:\|x\|<\beta_{1}\right\}, \overline{P_{\beta_{1}}}=\{x \in P:\|x\| \leq$ $\left.\beta_{1}\right\}, P\left(\rho, \beta_{1}, \beta_{4}\right)=\left\{x \in P: \beta_{1} \leq \rho(x),\|x\| \leq \beta_{4}\right\}$.

Now, we state the following Leggett-Williams fixed-point theorem, which is critical to the proof of our main results.

Lemma 2.1 (see [21]). Let $T: \overline{P_{\beta 4}} \rightarrow \overline{P_{\beta 4}}$ be completely continuous and $\rho$ nonnegative continuous concave functional on $P$ such that $\rho(u) \leq\|u\|$ for all $u \in \overline{P_{\beta 4}}$. Suppose that there exist positive constants $\beta_{1}, \beta_{2}, \beta_{3}, \beta_{4}$ with $0<\beta_{1}<\beta_{2}<\beta_{3} \leq \beta_{4}$ such that

(1) $\left\{u \in P\left(\rho, \beta_{2}, \beta_{3}\right): \rho(x)>\beta_{2}\right\} \neq \phi$ and $\rho(T u)>\beta_{2}$ for $u \in P\left(\rho, \beta_{2}, \beta_{3}\right)$;

(2) $\|T u\|<\beta_{1}$ for $u \in \overline{P_{\beta 1}}$;

(3) $\rho(T u)>\beta_{2}$ for $u \in P\left(\rho, \beta_{2}, \beta_{4}\right)$ with $\|T u\|>\beta_{3}$.

Then $T$ has at least three fixed points $u_{1}, u_{2}, u_{3}$ satisfying

$$
u_{1} \in P_{\beta 1}, \quad u_{2} \in\left\{u \in P\left(\rho, \beta_{2}, \beta_{4}\right): \rho(u)>\beta_{2}\right\}, \quad u_{3} \in \overline{P_{\beta 4}} \backslash\left(P\left(\rho, \beta_{2}, \beta_{4}\right) \cup \overline{P_{\beta 1}}\right) .
$$

Let $\mathbb{T}$ be a periodic time scale, and let $E=\{x \in C(\mathbb{T}, \mathbb{R}): x(t)=x(t+\omega)\}$ be a Banach space with the norm $\|x\|=\sup _{t \in[0, \omega] \cap \mathbb{T}}\{|x(t)|: x \in E\}$, and let $\Phi: E \rightarrow E$ be defined by

$$
(\Phi x)(t)=x(t)+c(t) x(t-\tau) .
$$


Lemma 2.2 (see [20]). If $0 \leq c(t)<1$ and $E$ is a Banach space, then $\Phi$ has a bounded inverse $\Phi^{-1}$ on $E$, and for all $x \in E$,

$$
\left(\Phi^{-1} x\right)(t)=\sum_{j \geq 0} \prod_{0 \leq i \leq j-1}(-1)^{k} c(t-i \tau) x(t-j \tau)
$$

and $\left\|\Phi^{-1} x\right\| \leq\|x\| /(1-\bar{c})$.

Definition 2.3 (see [6]). A nonempty closed set $\mathbb{T}_{0}(x)$ in $\mathbb{R} \times \mathbb{R}^{n}$ is said to be a variable time scale if for any $x_{0} \in \mathbb{R}^{n}$ the projection of $\mathbb{T}_{0}\left(x_{0}\right)$ on time axis, that is, the set $\left\{t \in \mathbb{R}:\left(t, x_{0}\right) \in \mathbb{T}_{0}\left(x_{0}\right)\right\}$ is a time scale in Hilger sense.

Fix a sequence $\left\{t_{i}\right\} \subset \mathbb{R}$ such that $t_{i}<t_{i+1}$ for all $i \in \mathbb{Z}$, and $\left|t_{i}\right| \rightarrow \infty$ as $|i| \rightarrow \infty$. Denote $\delta_{i}=t_{2 i-1}-t_{2 i}, \kappa_{i}=t_{2 i}-t_{2 i-1}$ and take a sequence of functions $\left\{\tau_{i}(x)\right\} \subset C\left(\mathbb{R}^{n}, \mathbb{R}\right)$. Assume that

$\left(C_{1}\right)$ for some positive numbers $\theta^{\prime}, \theta \in \mathbb{R}, \theta^{\prime} \leq t_{i+1}-t_{i} \leq \theta$;

$\left(C_{2}\right)$ there exists $l_{0}, 0<2 l_{0}<\theta^{\prime}$ such that $\left\|\tau_{i}(x)\right\| \leq l_{0}$ for all $x \in \mathbb{R}^{n}, i \in \mathbb{Z}$.

Denote

$$
l_{i}:=\inf _{x \in \mathbb{R}^{n}}\left\{t_{i}+\tau_{i}(x)\right\}, \quad r_{i}:=\sup _{x \in \mathbb{R}^{n}}\left\{t_{i}+\tau_{i}(x)\right\}
$$

We set

$$
\begin{aligned}
& \mathcal{E}_{i}=\left\{(t, x) \in \mathbb{R} \times \mathbb{R}^{n}: t_{2 i}+\tau_{2 i}(x)<t<t_{2 i+1}+\tau_{2 i+1}(x)\right\}, \\
& \mathcal{S}_{i}=\left\{(t, x) \in \mathbb{R} \times \mathbb{R}^{n}: t=t_{i}+\tau_{i}(x)\right\}, \\
& \boldsymbol{\Xi}_{i}=\left\{(t, x) \in \mathbb{R} \times \mathbb{R}^{n}: t_{2 i-1}+\tau_{2 i-1}(x) \leq t \leq t_{2 i}+\tau_{2 i}(x)\right\} .
\end{aligned}
$$

Following [6], we denote $\mathbb{T}_{0}(x):=\bigcup_{i=-\infty}^{\infty} \Phi_{i}$ and $\mathbb{T}_{c}=\bigcup_{i=-\infty}^{\infty}\left[t_{2 i-1}, t_{2 i}\right]$.

A transition operator $\Pi_{i}: \mathcal{S}_{2 i} \rightarrow \mathcal{S}_{2 i+1}$, for all $i \in \mathbb{Z}$, such that $\Pi_{i}(t, y)=$ $\left(\Pi_{i}^{1}(t, y), \Pi_{i}^{2}(t, y)\right)$ where $\Pi_{i}^{1}: \mathcal{S}_{2 i} \rightarrow \mathbb{R}$ and $\Pi_{i}^{2}: \mathcal{S}_{2 i} \rightarrow \mathbb{R}^{n}$, and

$$
\Pi_{i}^{1}(t, y)=t_{2 i+1}+\tau_{2 i+1}\left(\Pi_{i}^{2}(t, y)\right), \quad \Pi_{i}^{2}(t, y)=I_{i}(y)+y
$$

where $I_{i}: \mathbb{R}^{n} \rightarrow \mathbb{R}^{n}$ is a function. One can easily see that $\Pi_{i}^{1}(t, y)$ is the time coordinate of $\left(t^{+}, y^{+}\right):=\Pi_{i}(t, y)$, the image of $(t, y) \in \mathcal{S}_{2 i}$ under the operator $\Pi_{i}$, and $\Pi_{i}^{2}(t, y)$ is the space coordinate of the image.

Let $t=\alpha_{i}$ and $t=\beta_{i}$ be the moments that the graph of $y=\varphi(t)$ intersects the surface $\mathcal{S}_{2 i-1}$ and $\mathcal{S}_{2 i}$, respectively, where the surfaces are defined previously. Then, we set the nonvariable time scale

$$
\mathbb{T}_{c}^{\varphi}:=\bigcup_{i=-\infty}^{\infty}\left[\alpha_{i}, \beta_{i}\right]
$$


which is the domain of $\varphi$, and define the $\Delta$-derivative as in the introduction. That is, for $t=\beta_{i}$, we have

$$
\begin{aligned}
\varphi^{\Delta}\left(\beta_{i}\right) & =\frac{\varphi\left(\alpha_{i+1}\right)-\varphi\left(\beta_{i}\right)}{\alpha_{i+1}-\beta_{i}}, \\
\varphi^{\Delta}(t) & =\lim _{s \rightarrow t} \frac{\varphi(s)-\varphi(t)}{s-t}
\end{aligned}
$$

for any other $t \in \mathbb{T}_{c}^{\varphi}$, whenever the limit exists.

Consider the system on variable time scales:

$$
\begin{gathered}
y^{\prime}=A(t) y+f(t, y), \quad(t, y) \in \mathbb{T}_{0}(y), \\
\left.\Delta t\right|_{(t, y) \in \mathcal{S}_{2 i}}=\Pi_{i}^{1}(t, y)-t, \\
\left.\Delta y\right|_{(t, y) \in \mathcal{S}_{2 i}}=\Pi_{i}^{2}(t, y)-y,
\end{gathered}
$$

where $A(t): \mathbb{R} \rightarrow \mathbb{R}^{n \times n}$ is an $n \times n$ continuous real-valued matrix function, $B_{i}$ is an $n \times n$ matrix, functions $f(t, y): \mathbb{T}_{0}(y) \rightarrow \mathbb{R}^{n}$ and $J_{i}(y): \mathbb{R}^{n} \rightarrow \mathbb{R}^{n}$ are continuous, $\Pi_{i}^{1}(t, y)=$ $t_{2 i+1}+\tau_{2 i+1}\left(\Pi_{i}^{2}(t, y)\right)$, and $\Pi_{i}^{2}(t, y)=B_{i} y+J_{i}(y)+y$.

For any $\alpha, \beta \in \mathbb{R}$ we define the oriented interval $[\widehat{\alpha, \beta}]$ as

$$
[\widehat{\alpha, \beta}]= \begin{cases}{[\alpha, \beta],} & \text { if } \alpha \leq \beta \\ {[\beta, \alpha],} & \text { otherwise. }\end{cases}
$$

Consider the nonvariable time scale

$$
\mathbb{T}_{c}^{0}=\bigcup_{i=-\infty}^{\infty}\left[l_{2 i-1}, r_{2 i}\right]
$$

where $l_{i}, r_{i}, i \in \mathbb{Z}$ are defined by (2.5) for the variable time scale $\mathbb{T}_{0}(y)$, and take a continuation $\tilde{f}: \mathbb{T}_{c}^{0} \times \mathbb{R}^{n} \rightarrow \mathbb{R}^{n}$ of $f: \mathbb{T}_{0}(y) \rightarrow \mathbb{R}^{n}$ which is Lipschitzian with the same Lipschitz constant $l$; furthermore, if $f$ is a monotone function, a continuation $\tilde{f}$ can also have the same monotony with $f$. Set $\mathbb{T}_{c}:=\bigcup_{i=-\infty}^{\infty}\left[t_{2 i-1}, t_{2 i}\right]$.

$\left(C_{3}\right)\left\|\tau_{i}(x)-\tau_{i}(y)\right\|+\left\|J_{i}(x)-J_{i}(y)\right\|+\|f(t, x)-f(t, y)\| \leq l\|x-y\|$ for arbitrary $x, y \in \mathbb{R}^{n}$, where $l$ is a Lipschitz constant.

By $\left(C_{3}\right)$, in [6], one can see the following important lemma.

Lemma 2.4 (see [6]). Assume $\left(C_{3}\right)$ is satisfied. Then there are mappings $W_{i}(z): \mathbb{R}^{n} \rightarrow \mathbb{R}^{n}, i \in \mathbb{Z}$, such that, corresponding to each solution $y(t)$ of (2.10), there is a solution $z(t)$ of the system

$$
\begin{gathered}
z^{\prime}=A(t) z+\tilde{f}(t, z), \quad t \neq t_{2 i}, \\
z\left(t_{2 i+1}\right)=B_{i} z\left(t_{2 i}\right)+W_{i}\left(z\left(t_{2 i}\right)\right)+z\left(t_{2 i}\right),
\end{gathered}
$$


such that $y(t)=z(t)$ for all $t \in \mathbb{T}_{c}$ except possibly on $\left[\widehat{\left[t_{2 i-1}, \alpha_{i}\right.}\right]$ and $\left[\widehat{\beta_{i}, t_{2 i}}\right]$, where $\alpha_{i}$ and $\beta_{i}$ are the moments that $y(t)$ meets the surfaces $\mathcal{S}_{2 i-1}$ and $\mathcal{S}_{2 i}$, respectively.

Furthermore, the functions $W_{i}$ satisfy the inequality

$$
\left\|W_{i}(z)-W_{i}(y)\right\| \leq k(l) l\|z-y\|,
$$

uniformly with respect to $i \in \mathbb{Z}$ for all $z, y \in \mathbb{R}^{n}$ such that $\|z\| \leq h$ and $\|y\| \leq h$; here $k\left(l_{0}\right)=k\left(l_{0}, h\right)$ is a bounded function. Under the sense of Lemma 2.4, we say that systems (2.10) and (2.13) are Bequivalent.

Proof. Fix $i \in \mathbb{Z}$. Let $z(t)$ be the solution of (2.10) such that $z\left(t_{2 i}\right)=z$, and assume that $\alpha_{\mathrm{i}}$ and $\beta_{i}$ are solutions of $\alpha=t_{2 i-1}+\tau_{2 i-1}(z(\alpha))$ and $\beta=t_{2 i}+\tau_{2 i}(z(\beta))$, respectively. Let $z_{1}(t)$ be the solution of the system

$$
z^{\prime}=A(t) z+\tilde{f}(t, z)
$$

with the initial condition $z_{1}\left(\alpha_{i+1}\right)=\Pi_{i}^{2}\left(\beta_{i}, z\left(\beta_{i}\right)\right)$.

We first note that $z_{1}\left(\alpha_{i+1}\right)=\left(I+B_{i}\right) z\left(\beta_{i}\right)+J_{i}\left(z\left(\beta_{i}\right)\right)$. Moreover, for $t \in\left[\widehat{t_{2 i}, \beta_{i}}\right]$,

$$
z(t)=z\left(t_{2 i}\right)+\int_{t_{2 i}}^{t}[A(s) z(s)+\tilde{f}(s, z(s))] \mathrm{d} s,
$$

and for $t \in\left[\widehat{\alpha_{i+1}, t_{2 i+1}}\right]$,

$$
\begin{aligned}
z_{1}(t)= & z_{1}\left(\alpha_{i+1}\right)+\int_{\alpha_{i+1}}^{t}\left[A(s) z_{1}(s)+f\left(s, z_{1}(s)\right)\right] \mathrm{d} s \\
= & \left(I+B_{i}\right) z\left(\beta_{i}\right)+J_{i}\left(z\left(\beta_{i}\right)\right)+\int_{\alpha_{i+1}}^{t}\left[A(s) z_{1}(s)+\tilde{f}\left(s, z_{1}(s)\right)\right] \mathrm{d} s \\
= & \left(I+B_{i}\right)\left[z\left(t_{2 i}\right)+\int_{t_{2 i}}^{\beta_{i}}[A(s) z(s)+\tilde{f}(s, z(s))] \mathrm{d} s\right]+J_{i}\left(z\left(\beta_{i}\right)\right) \\
& +\int_{\alpha_{i+1}}^{t}\left[A(s) z_{1}(s)+\tilde{f}\left(s, z_{1}(s)\right)\right] \mathrm{d} s .
\end{aligned}
$$

Thus, we have

$$
\begin{aligned}
W_{i}(z)= & \left(I+B_{i}\right) \int_{t_{2 i}}^{\beta_{i}}[A(s) z(s)+\tilde{f}(s, z(s))] \mathrm{d} s+J_{i}\left(z\left(\beta_{i}\right)\right) \\
& +\int_{\alpha_{i+1}}^{t_{2 i+1}}\left[A(s) z_{1}(s)+\tilde{f}\left(s, z_{1}(s)\right)\right] \mathrm{d} s .
\end{aligned}
$$

Substituting (2.18) in (2.13), we see that $W_{i}(z)$ satisfies the first conclusion of the lemma. 
Next, we prove (2.14). Let $\left\|z\left(t_{2 i}\right)\right\| \leq h$. By employ integrals (2.16) and (2.17), we find that the solutions $z(t)$ and $z_{1}(t)$ determined above satisfy the inequalities $\|z(t)\| \leq H$ and $\left\|z_{1}(t)\right\| \leq H$ on $\left[\widehat{\beta_{i}, t_{2 i}}\right]$ and $\left[\alpha_{i+1}, t_{2 i+1}\right]$, where

$$
H=\left[M(1+l)+(1+N+l)(h+M l) e^{N l+l^{2}}\right] e^{N l+l^{2}} .
$$

Let $y(t)$ be the solution of (2.10) such that $y\left(t_{2 i}\right)=y$, and assume that $\bar{\alpha}_{i}$ and $\bar{\beta}_{i}$ are solutions of $\bar{\alpha}=t_{2 i-1}+\tau_{2 i-1}(y(\bar{\alpha}))$ and $\bar{\beta}=t_{2 i}+\tau_{2 i}(y(\bar{\beta}))$, respectively. Let $y_{1}(t)$ be the solution of (2.15) with initial condition $y_{1}\left(\bar{\alpha}_{i+1}\right)=\Pi_{i}^{2}\left(\bar{\beta}_{i}, y\left(\bar{\beta}_{i}\right)\right)$. Without loss of any generality, we assume that $\bar{\beta}_{i} \geq \beta_{i}$ and $\bar{\alpha}_{i+1} \leq \alpha_{i+1}$. Application of the Gronwall-Bellman lemma shows that, for $t \in\left[\widehat{\beta_{i}, t_{2 i}}\right]$,

$$
\|z(t)-y(t)\| \leq e^{(N+l) l}\|z-y\| .
$$

The equation

$$
y\left(\bar{\beta}_{i}\right)=y\left(\beta_{i}\right)+\int_{\beta_{i}}^{\bar{\beta}_{i}}[A(s) y(s)+\tilde{f}(s, y(s))] \mathrm{d} s
$$

gives us

$$
\left\|y\left(\bar{\beta}_{i}\right)-y\left(\beta_{i}\right)\right\| \leq(N H+l H+M)\left(\bar{\beta}_{i}-\beta_{i}\right)
$$

Thus, we obtain

$$
\left\|z\left(\beta_{i}\right)-y\left(\bar{\beta}_{i}\right)\right\| \leq e^{(N+l) l}\|z-y\|+(N H+l H+M)\left(\bar{\beta}_{i}-\beta_{i}\right) .
$$

Now condition $\left(C_{3}\right)$ together with (2.23) leads to

$$
\bar{\beta}_{i}-\beta_{i} \leq \frac{l e^{(N+l) l}}{1-l(N H+l H+M)}\|z-y\|
$$

Hence (2.23) becomes

$$
\left\|z\left(\beta_{i}\right)-y\left(\bar{\beta}_{i}\right)\right\| \leq \frac{e^{(N+l) l}}{1-l(N H+l H+M)}\|z-y\| .
$$

On the other hand,

$$
y_{1}\left(\alpha_{i+1}\right)=y_{1}\left(\bar{\alpha}_{i+1}\right)+\int_{\bar{\alpha}_{i+1}}^{\alpha_{i+1}}\left[A(s) y_{1}(s)+\tilde{f}\left(s, y_{1}(s)\right)\right] \mathrm{d} s
$$


gives us

$$
\left\|y_{1}\left(\alpha_{i+1}\right)-y_{1}\left(\bar{\alpha}_{i+1}\right)\right\| \leq(N H+l H+M)\left(\alpha_{i+1}-\bar{\alpha}_{i+1}\right) .
$$

Using the transition operators and (2.25) we get

$$
\left\|z_{1}\left(\alpha_{i+1}\right)-y_{1}\left(\bar{\alpha}_{i+1}\right)\right\| \leq \frac{(1+N+l) e^{(N+l) l}}{1-l(N H+l H+M)}\|z-y\| .
$$

Condition $\left(\mathrm{C}_{3}\right)$ and (2.28) imply that

$$
\alpha_{i+1}-\bar{\alpha}_{i+1} \leq \frac{l(1+N+l) e^{(N+l) l}}{1-l(N H+l H+M)}\|z-y\|
$$

From (2.27)-(2.29) we obtain

$$
\left\|z_{1}\left(\alpha_{i+1}\right)-y_{1}\left(\bar{\alpha}_{i+1}\right)\right\| \leq H_{1} e^{(N+l) l}\|z-y\|,
$$

where $H_{1}=(1+N+l)[1+l(N H+l H+M)] /[1-l(N H+l H+M)]$. Solutions $z_{1}(t)$ and $y_{1}(t)$ on $\left[\widehat{\alpha_{i+1}, t_{2 i+1}}\right]$ satisfy the inequality

$$
\left\|z_{1}(t)-y_{1}(t)\right\| \leq H_{1} e^{2(N+l) l}\|z-y\|
$$

Now subtracting the expression

$$
\begin{aligned}
W_{i}(y)= & \left(I+B_{i}\right) \int_{t_{2 i}}^{\bar{\beta}_{i}}[A(s) y(s)+\tilde{f}(s, y(s))] \mathrm{d} s+J_{i}\left(y\left(\bar{\beta}_{i}\right)\right) \\
& +\int_{\bar{\alpha}_{i+1}}^{t_{2 i+1}}\left[A(s) y_{1}(s)+\tilde{f}\left(s, y_{1}(s)\right)\right] \mathrm{d} s
\end{aligned}
$$

from (2.18) and using (2.20), (2.24), (2.29), and (2.31), we conclude that (2.14) holds. The proof is complete.

A special transformation called $\psi$-substitution [5], which is change of the independent variable and defined for $t \in \bigcup_{i=-\infty}^{\infty}\left(t_{2 i-1}, t_{2 i}\right]$ as

$$
\psi(t)= \begin{cases}t-\sum_{0<t_{2 k}<t} \delta_{k}, & t \geq 0 \\ t+\sum_{t \leq t_{2 k}<0} \delta_{k}, & t<0\end{cases}
$$


where $\delta_{k}=t_{2 k+1}-t_{2 k}$. Setting $s_{i}=\psi\left(t_{2 i}\right)$, we see that this transformation has an inverse given by

$$
\psi^{-1}(s)= \begin{cases}s+\sum_{0<s_{k}<s} \delta_{k}, & s \geq 0, \\ s-\sum_{s \leq s_{k}<0} \delta_{k}, & s<0 .\end{cases}
$$

Lemma 2.5 (see $[5,6]) . \psi^{\prime}(t)=1$ if $t \in \bigcup_{i=-\infty}^{\infty}\left(t_{2 i-1}, t_{2 i}\right]$.

Proof. Assume that $t \geq 0$. Then,

$$
\begin{aligned}
\psi^{\prime}(t) & =\lim _{h \rightarrow 0} \frac{\psi(t+h)-\psi(t)}{h} \\
& =\lim _{h \rightarrow 0} \frac{1}{h}\left[\left(t+h-\sum_{0<t_{2 k}<t+h} \delta_{k}\right)-\left(t-\sum_{0<t_{2 k}<t} \delta_{k}\right)\right]=1 .
\end{aligned}
$$

The assertion for $t<0$ can be proved in the same way. The proof is complete.

Definition 2.6 (see [5]). The time scale $\mathbb{T}_{0}$ is said to have an $\omega$-property if there exists a number $\omega \in \mathbb{R}^{+}$such that $t+\omega \in \mathbb{T}_{0}$ whenever $t \in \mathbb{T}_{0}$.

Definition 2.7 (see [5]). A sequence $\left\{a_{i}\right\} \subset \mathbb{R}$ is said to satisfy an $(\omega, p)$-property if there exist numbers $\omega \in \mathbb{R}^{+}$and $p \in \mathbb{N}$ such that $a_{i+p}=a_{i}+\omega$ for all $i \in \mathbb{Z}$.

Definition 2.8 (see [6]). The variable time scale $\mathbb{T}_{0}(y)$ is said to satisfy an $(\omega, p)$-property if $(t \pm \omega, y)$ is in $\mathbb{T}_{0}(y)$ whenever $(t, y)$ is. In this case, there exists $p \in \mathbb{N}$ such that the sequences $\left\{t_{2 i-1}\right\}$ and $\left\{t_{2 i}\right\}$ satisfy the $(\omega, p)$-property and $\tau_{i+p}(y)=\tau_{i}(y)$ for all $i \in \mathbb{Z}$.

Suppose now that $(2.10)$ is $\omega$-periodic; that is, $\mathbb{T}_{0}(y)$ satisfies the $(\omega, p)$-property, $A(t)$ and $f(t, y)$ are $\omega$-periodic functions of $t$, and $B_{i+p}=B_{i}, J_{i+p}(y)=J_{i}(y)$ uniformly with respect to $i \in \mathbb{Z}$.

Lemma 2.9 (see [6]). If (2.10) is w-periodic, then the sequence $W_{i}(z)$ is p-periodic uniformly with respect to $z \in \mathbb{R}^{n}$.

Proof. Since the variable time scale $\mathbb{T}_{0}(y)$ satisfies an $(\omega, p)$-property, by $(2.18)$, one can easily see that $W_{i}(z)$ is $p$-periodic uniformly with respect to $z \in \mathbb{R}^{n}$. The proof is complete.

Lemma 2.10 (see [5]). If $\mathbb{T}_{0}$ has an w-property, then the sequence $\left\{s_{i}\right\}, s_{i}=\psi\left(t_{2 i}\right)$, is $\left(\tilde{\omega}, p_{0}\right)$ periodic with

$$
\tilde{\omega}=\omega-\sum_{0<t_{2 k}<\omega} \delta_{k}=\psi(\omega) .
$$


Proof. In order to prove this lemma, we only need to verify that $s_{i+p_{0}}=s_{i}+\tilde{\omega}$ for all $i$. Assume that $i \geq 0, i=n p_{0}+j$ for some $n \in \mathbb{Z}, 0 \leq j<p_{0}$ and $0<t_{0}<\cdots<t_{2\left(p_{0}-1\right)}<\omega$. Then

$$
\begin{aligned}
s_{i+p_{0}} & =\psi\left(t_{2\left(i+p_{0}\right)}\right)=t_{2\left(i+p_{0}\right)}-\sum_{0<t_{2 k}<t_{2\left(i+p_{0}\right)}} \delta_{k} \\
& =t_{2 i}+\omega-\sum_{0<t_{2 k}<t_{2 i}} \delta_{k}-\sum_{t_{2 i} \leq t_{2 k}<t_{2\left(i+p_{0}\right)}} \delta_{k}=\psi\left(t_{2 i}\right)+\omega-\sum_{k=i}^{j+p_{0}-1} \delta_{k} \\
& =s_{i}+\omega-\sum_{k=j}^{j+p_{0}-1} \delta_{k+n p_{0}}=s_{i}+\omega-\sum_{k=j}^{j+p_{0}-1} \delta_{k}=s_{i}+\omega-\sum_{k=0}^{p_{0}-1} \delta_{k} \\
& =s_{i}+\omega-\sum_{0<t_{2 k}<\omega} \delta_{k}=s_{i}+\tilde{\omega},
\end{aligned}
$$

where we have used the fact that

$$
\begin{aligned}
\sum_{k=j}^{j+p_{0}-1} \delta_{k} & =\sum_{k=j}^{p_{0}-1} \delta_{k}+\sum_{k=p_{0}}^{j+p_{0}-1} \delta_{k}=\sum_{k=j}^{p_{0}-1} \delta_{k}+\sum_{k=0}^{j-1} \delta_{k+p_{0}} \\
& =\sum_{k=j}^{p_{0}-1} \delta_{k}+\sum_{k=0}^{j-1} \delta_{k}=\sum_{k=0}^{p_{0}-1} \delta_{k} .
\end{aligned}
$$

All other cases can be verified similarly. The proof is complete.

Lemma 2.11 (see $[5,6])$. If $\mathbb{T}_{0}(y)$ satisfies an $(\omega, p)$-property, then $\psi(t+\omega)=\psi(t)+\psi(\omega)$.

Proof. Assume that $t \geq 0$. By Lemma 2.10, we have

$$
\begin{aligned}
\psi(t+\omega) & =t+\omega-\sum_{0<t_{2 k}<t+\omega} \delta_{k}=t+\omega-\sum_{0<t_{2 k}<\omega} \delta_{k}-\sum_{\omega \leq t_{2 k}<t+\omega} \delta_{k} \\
& =t-\sum_{\omega \leq t_{2 k}<t+\omega} \delta_{k}+\psi(\omega)=\psi(t)+\psi(\omega) .
\end{aligned}
$$

The assertion for $t<0$ can be proved in the same way. The proof is complete.

Lemma 2.12 (see $[5,6])$. A function $\phi(t)$ is an $\omega$-periodic function on $\mathbb{T}_{c}$, if and only if $\phi\left(\psi^{-1}(s)\right)$ is an $\tilde{\omega}$-periodic function on $\mathbb{R}$, where $\widetilde{\omega}=\psi(\omega)$.

Proof. By Lemma 2.11, $s+\tilde{\omega}=\psi(t+\omega)$. Then the equality

$$
\phi\left(\psi^{-1}(s+\tilde{\omega})\right)=\phi(t+\omega)=\phi(t)=\phi\left(\psi^{-1}(s)\right)
$$

completes the proof.

For any fixed $x_{0} \in \mathbb{R}$, we set 
Journal of Applied Mathematics

$\begin{aligned} \operatorname{PC}( & \left.\mathbb{T}\left(x_{0}\right)\right) \\ \quad & =\left\{x \in C\left(\left(t_{2 i-1}+\tau_{2 i-1}\left(x_{0}\right), t_{2 i}+\tau_{2 i}\left(x_{0}\right)\right), \mathbb{R}\right): \Pi_{i}^{2}(t, x)=B_{i} x+J_{i}(x)+x, i=1,2, \ldots\right\}\end{aligned}$

and consider the Banach space

$$
E=\left\{x: x \in P C\left(\mathbb{T}\left(x_{0}\right)\right): x(t)=x(t+\omega)\right\} .
$$

Let $\Phi: E \rightarrow E$ be defined by

$$
(\Phi x)(t)=x(t)+c(t) x(t-\alpha):=y(t) .
$$

Using the inverse transformation of $\Phi$, we can obtain

$$
\Pi_{i}^{1}\left(t, \Phi^{-1} y\right)=t_{2 i+1}+\tau_{2 i+1}\left(\Pi_{i}^{2}\left(t, \Phi^{-1} y\right)\right), \quad \Pi_{i}^{2}\left(t, \Phi^{-1} y\right)=B_{i} \Phi^{-1} y+J_{i}\left(\Phi^{-1} y\right)+\Phi^{-1} y
$$

From the second equation, it is easy to get

$$
\Phi\left(\Pi_{i}^{2}\left(t, \Phi^{-1} y\right)\right)=\left(\Phi B_{i} \Phi^{-1}\right) y+\left(\Phi J_{i} \Phi^{-1}\right) y+y:=\widetilde{\Pi}_{i}^{2}(t, y),
$$

that is, $\Pi_{i}^{2}\left(t, \Phi^{-1} y\right)=\Phi^{-1}\left(\widetilde{\Pi}_{i}^{2}(t, y)\right)$. Hence

$$
\begin{aligned}
\Pi_{i}^{1}\left(t, \Phi^{-1} y\right) & =t_{2 i+1}+\tau_{2 i+1}\left(\Phi^{-1} \tilde{\Pi}_{i}^{2}(t, y)\right)=t_{2 i+1}+\tau_{2 i+1} \Phi^{-1}\left(\widetilde{\Pi}_{i}^{2}(t, y)\right) \\
& :=t_{2 i+1}+\widetilde{\tau}_{2 i+1}\left(\tilde{\Pi}_{i}^{2}(t, y)\right):=\tilde{\Pi}_{i}^{1}(t, y) .
\end{aligned}
$$

Therefore, we can obtain the other variable time scale plane $T_{0}(y)$ by the inverse transformation of $\Phi$ and

$$
\begin{aligned}
& \mathcal{E}_{i}^{\prime}=\left\{(t, y) \in \mathbb{R} \times \mathbb{R}: t_{2 i}+\widetilde{\tau}_{2 i}(y)<t<t_{2 i+1}+\widetilde{\tau}_{2 i+1}(y)\right\}, \\
& \mathcal{S}_{i}^{\prime}=\left\{(t, y) \in \mathbb{R} \times \mathbb{R}: t=t_{i}+\widetilde{\tau}_{i}(y)\right\}, \\
& \boldsymbol{\Phi}_{i}^{\prime}=\left\{(t, y) \in \mathbb{R} \times \mathbb{R}: t_{2 i-1}+\widetilde{\tau}_{2 i-1}(y) \leq t \leq t_{2 i}+\widetilde{\tau}_{2 i}(y)\right\} .
\end{aligned}
$$

Hence, (1.2) can be changed into the following form:

$$
\begin{gathered}
y^{\prime}=a(t) g\left(\left(\Phi^{-1} y\right)(t)\right) y(t)-a(t) H(y(t))-\sum_{j=1}^{n} \lambda_{j} f_{j}\left(t,\left(\Phi^{-1} y\right)\left(t-v_{j}(t)\right)\right), \quad(t, y) \in \mathbb{T}_{0}(y), \\
\left.\Delta t\right|_{(t, y) \in \mathcal{S}_{2 i}^{\prime}}=\widetilde{\Pi}_{i}^{1}(t, y)-t, \\
\left.\Delta y\right|_{(t, y) \in \mathcal{S}_{2 i}^{\prime}}=\widetilde{\Pi}_{i}^{2}(t, y)-y,
\end{gathered}
$$

where $H(y(t))=c(t) g\left(\left(\Phi^{-1} y\right)(t)\right)\left(\Phi^{-1} y\right)(t-\alpha)$. 
Define a cone in $E$ by

$$
P_{0}=\{y(t) \in E: y(t) \geq k\|y\|\},
$$

where $k \in\left(\left((2 \delta-\underline{c})\left(1-\underline{c}^{2}\right)\right) /\left(1-(2 \delta-\underline{c})^{2}\right), r_{0}^{L}\left(1-r_{0}^{l}\right) /\left(1-r_{0}^{L}\right)\right]$.

Lemma 2.13 (see [20]). Suppose that conditions $\left(H_{1}\right)-\left(H_{4}\right)$ hold and $0 \leq c(t)<1$ and $y \in P_{0}$, then

$$
\begin{aligned}
& \tilde{\alpha}\|y\| \leq\left(\Phi^{-1} y\right)(t) \leq \frac{1}{1-\bar{c}}\|y\|, \\
& l_{\underline{c} \tilde{\alpha}}\|y\| \leq H(y(t)) \leq \frac{L \bar{c}}{1-\bar{c}}\|y\|,
\end{aligned}
$$

where $\tilde{\alpha}=\left(k /\left(1-\underline{c}^{2}\right)\right)-(2 \delta-\underline{c}) /\left(1-(2 \delta-\underline{c})^{2}\right)$.

$\left(\mathrm{H}_{5}\right)\left\|\tau_{i}(x)-\tau_{i}(y)\right\|+\left\|J_{i}(x)-J_{i}(y)\right\|+\sum_{j=1}^{n}\left\|f_{j}(t, x)-f_{j}(t, y)\right\| \leq l_{0}\|x-y\|$ for arbitrary $x, y \in \mathbb{R}^{n}$, where $l_{0}$ is a Lipschitz constant.

In view of $\left(\mathrm{H}_{5}\right)$ by Lemma 2.4 , it is easy to get the following lemma.

Lemma 2.14. Assume that $\left(H_{5}\right)$ is satisfied. Then there are mappings $W_{i}(z): \mathbb{R} \rightarrow \mathbb{R}, i \in \mathbb{Z}$ such that, corresponding to each solution $y(t)$ of (2.48), there is a solution $z(t)$ of the system

$$
\begin{gathered}
z^{\prime}=a(t) g\left(\left(\Phi^{-1} z\right)(t)\right) z(t)-a(t) H(z(t))-\sum_{j=1}^{n} \lambda_{j} \tilde{f}_{j}\left(t,\left(\Phi^{-1} z\right)\left(t-v_{j}(t)\right)\right), \quad t \neq t_{2 i}, \\
z\left(t_{2 i+1}\right)=\left(\Phi B_{i} \Phi^{-1}\right)\left(z\left(t_{2 i}\right)\right)+\left(\Phi W_{i} \Phi^{-1}\right)\left(z\left(t_{2 i}\right)\right)+z\left(t_{2 i}\right), \quad t=t_{2 i}
\end{gathered}
$$

such that $y(t)=z(t)$ for all $t \in \mathbb{T}_{c}$ except possibly on $\left[\widehat{t_{2 i-1}, \alpha_{i}}\right]$ and $\left[\widehat{\beta_{i}, t_{2 i}}\right]$ where $\alpha_{i}$ and $\beta_{i}$ are the moments that $y(t)$ meets the surfaces $\mathcal{S}_{2 i-1}^{\prime}$ and $\mathcal{S}_{2 i}^{\prime}$, respectively.

Furthermore, the functions $W_{i}$ satisfy the inequality

$$
\left\|W_{i}(z)-W_{i}(y)\right\| \leq k\left(l_{0}\right) l_{0}\|z-y\|
$$

uniformly with respect to $i \in \mathbb{Z}$ for all $z, y \in \mathbb{R}^{n}$ such that $\|z\| \leq h$ and $\|y\| \leq h$; here $k\left(l_{0}\right)=k\left(l_{0}, h\right)$ is a bounded function. Under the sense of lemma 2.14, we say that systems (2.48) and (2.52) are B-equivalent.

Proof. For fixed $i \in \mathbb{Z}$. Let $z(t)$ be the solution of (2.48) such that $z\left(t_{2 i}\right)=z$, and assume that $\alpha_{i}$ and $\beta_{i}$ are solutions of $\alpha=t_{2 i-1}+\widetilde{\tau}_{2 i-1}(z(\alpha))$ and $\beta=t_{2 i}+\widetilde{\tau}_{2 i}(z(\beta))$, respectively. Let $z_{1}(t)$ be the solution of the system

$$
z^{\prime}=a(t) g\left(\left(\Phi^{-1} z\right)(t)\right) z(t)-a(t) H(z(t))-\sum_{j=1}^{n} \lambda_{j} \tilde{f}_{j}\left(t,\left(\Phi^{-1} z\right)\left(t-v_{j}(t)\right)\right)
$$

with the initial condition $z_{1}\left(\alpha_{i+1}\right)=\tilde{\Pi}_{i}^{2}\left(\beta_{i}, z\left(\beta_{i}\right)\right)$. 

$t \in\left[\widehat{t_{2 i}, \beta_{i}}\right]$,

We first note that $z_{1}\left(\alpha_{i+1}\right)=\left(\Phi B_{i} \Phi^{-1}\right)\left(z\left(\beta_{i}\right)\right)+\left(\Phi J_{i} \Phi^{-1}\right)\left(z\left(\beta_{i}\right)\right)+z\left(\beta_{i}\right)$. Moreover, for

$$
\begin{aligned}
z(t)=z\left(t_{2 i}\right)+\int_{2 i}^{t} & {\left[a(s) g\left(\left(\Phi^{-1} z\right)(s)\right) z(s)-a(s) H(z(s))\right.} \\
& \left.-\sum_{j=1}^{n} \lambda_{j} \tilde{f}_{j}\left(s,\left(\Phi^{-1} z\right)\left(s-v_{j}(s)\right)\right)\right] \mathrm{d} s,
\end{aligned}
$$

and for $t \in\left[\widehat{\alpha_{i+1}, t_{2 i+1}}\right]$,

$$
\begin{aligned}
z_{1}(t)= & z_{1}\left(\alpha_{i+1}\right)+\int_{\alpha_{i+1}}^{t}\left[a(s) g\left(\left(\Phi^{-1} z_{1}\right)(s)\right) z_{1}(s)\right. \\
& \left.-a(s) H\left(z_{1}(s)\right)-\sum_{j=1}^{n} \lambda_{j} \tilde{f}_{j}\left(s,\left(\Phi^{-1} z_{1}\right)\left(s-v_{j}(s)\right)\right)\right] \mathrm{d} s \\
= & \left(\Phi B_{i} \Phi^{-1}\right)\left(z\left(\beta_{i}\right)\right)+\left(\Phi J_{i} \Phi^{-1}\right)\left(z\left(\beta_{i}\right)\right)+z\left(\beta_{i}\right) \\
& +\int_{\alpha_{i+1}}^{t}\left[a(s) g\left(\left(\Phi^{-1} z_{1}\right)(s)\right) z_{1}(s)\right. \\
= & \left(\Phi\left(B_{i}+I\right) \Phi^{-1}\right)\left[z\left(t_{2 i}\right)+\int_{t_{2 i}}^{\beta_{i}}\left[a(s) g\left(\left(\Phi^{-1} z\right)(s)\right) z(s)-a(s) H(z(s))\right.\right. \\
& \left.\left.-\sum_{j=1}^{n} \lambda_{j} \tilde{f}_{j}\left(s,\left(\Phi^{-1} z\right)\left(s-v_{j}(s)\right)\right)\right] \mathrm{d} s\right] \\
& +\left(\Phi J_{i} \Phi^{-1}\right)\left(z\left(\beta_{i}\right)\right)+\int_{\alpha_{i+1}}^{t}\left[a(s) g\left(\left(\Phi^{-1} z_{1}\right)(s)\right) z_{1}(s)\right. \\
& \left.-a(s) H\left(z_{1}(s)\right)-\sum_{j=1}^{n} \lambda_{j} \tilde{f}_{j}\left(s,\left(\Phi^{-1} z_{1}\right)\left(s-v_{j}(s)\right)\right)\right] \mathrm{d} s .
\end{aligned}
$$

Thus, we set

$$
\begin{aligned}
W_{i}(z)=\left(\Phi\left(B_{i}+I\right) \Phi^{-1}\right) \int_{t_{2 i}}^{\beta_{i}} & {\left[a(s) g\left(\left(\Phi^{-1} z\right)(s)\right) z(s)-a(s) H(z(s))\right.} \\
& \left.-\sum_{j=1}^{n} \lambda_{j} \tilde{f}_{j}\left(s,\left(\Phi^{-1} z\right)\left(s-v_{j}(s)\right)\right)\right] \mathrm{d} s+\left(\Phi J_{i} \Phi^{-1}\right)\left(z\left(\beta_{i}\right)\right)
\end{aligned}
$$




$$
\begin{aligned}
+\int_{\alpha_{i+1}}^{t_{2 i+1}}[ & a(s) g\left(\left(\Phi^{-1} z_{1}\right)(s)\right) z_{1}(s) \\
& \left.-a(s) H\left(z_{1}(s)\right)-\sum_{j=1}^{n} \lambda_{j} \tilde{f}_{j}\left(s,\left(\Phi^{-1} z_{1}\right)\left(s-v_{j}(s)\right)\right)\right] \mathrm{d} s .
\end{aligned}
$$

Substituting (2.57) in (2.52), we see that $W_{i}(z)$ satisfies the first conclusion of the lemma.

The rest of the proof is similar to that of Lemma 2.4, and we can use Gronwall-Bellman lemma to show that $W_{i}(z)$ satisfies (2.53) and it will be omitted here. This completes the proof.

Next, we will use $\psi$-substitution, reducing (2.52) to an impulsive differential equation. Letting $m(s)=z\left(\psi^{-1}(s)\right)$, we obtain, for $t \neq t_{2 i}$,

$$
\begin{aligned}
\left(\Phi^{-1} z\right)\left(t-v_{j}(t)\right) & =\left(\Phi^{-1} z\right)\left(\psi^{-1}(s)-v_{j}\left(\psi^{-1}(s)\right)\right) \\
& =\left(\Phi^{-1} z\right)\left(\psi^{-1}\left(\psi\left(\psi^{-1}(s)-v_{j}\left(\psi^{-1}(s)\right)\right)\right)\right) \\
& =\left(\Phi^{-1} m\right)\left(\psi\left(\psi^{-1}(s)-v_{j}\left(\psi^{-1}(s)\right)\right)\right):=v(s),
\end{aligned}
$$

hence

$$
m^{\prime}=a\left(\psi^{-1}(s)\right) g\left(\left(\Phi^{-1} m\right)(s)\right) m(s)-a\left(\psi^{-1}(s)\right) H(m(s))-\sum_{j=1}^{n} \lambda_{j} \tilde{f}_{j}\left(\psi^{-1}(s), v(s)\right),
$$

and for $t=t_{2 i}$, we get

$$
m\left(s_{i}^{-}\right)=z\left(t_{2 i+1}\right)=\left(\Phi B_{i} \Phi^{-1}\right)\left(m\left(s_{i}\right)\right)+\left(\Phi W_{i} \Phi^{-1}\right)\left(m\left(s_{i}\right)\right)+m\left(s_{i}\right)
$$

Thus, the second equation in (2.23) leads to

$$
\left.\Delta m\right|_{s=s_{i}}=\left(\Phi B_{i} \Phi^{-1}\right)\left(m\left(s_{i}\right)\right)+\left(\Phi W_{i} \Phi^{-1}\right)\left(m\left(s_{i}\right)\right),
$$

where $\left.\Delta m\right|_{s=s_{i}}=m\left(s_{i}^{-}\right)-m\left(s_{i}\right)$. Hence, $m(s)$ is a solution of the impulsive differential equation:

$$
\begin{gathered}
m^{\prime}=a\left(\psi^{-1}(s)\right) g\left(\left(\Phi^{-1} m\right)(s)\right) m(s)-a\left(\psi^{-1}(s)\right) H(m(s))-\sum_{j=1}^{n} \lambda_{j} \tilde{f}_{j}\left(\psi^{-1}(s), v(s)\right), \quad s \neq s_{i}, \\
\left.\Delta m\right|_{s=s_{i}}=\left(\Phi B_{i} \Phi^{-1}\right)\left(m\left(s_{i}\right)\right)+\left(\Phi W_{i} \Phi^{-1}\right)\left(m\left(s_{i}\right)\right), \quad s=s_{i} .
\end{gathered}
$$


Journal of Applied Mathematics

In the following, we set

$$
\overline{P C}=\left\{m:\left.m\right|_{\left(s_{i}, s_{i+1}\right)} \in C\left(\left(s_{i}, s_{i+1}\right), \mathbb{R}\right), m\left(s_{i}^{+}\right)=m\left(s_{i}\right), i=1,2, \ldots\right\}
$$

and consider the Banach space

$$
\bar{E}=\{m: m \in \overline{P C}, m(s)=m(s+\tilde{\omega})\}
$$

with the norm $\|m\|=\sup _{s \in[0, \tilde{\omega}]}\{|m(s)|: m \in \bar{E}\}$, where $\tilde{\omega}=\psi(\omega)$. Define a cone in $\bar{E}$ by

$$
P=\{m(s) \in \bar{E}: m(s) \geq k\|m\|\}
$$

where $k \in\left((2 \delta-\underline{c})\left(1-\underline{c}^{2}\right) /\left(1-(2 \delta-\underline{c})^{2}\right), r_{0}^{L}\left(1-r_{0}^{l}\right) /\left(1-r_{0}^{L}\right)\right]$.

Let the operator $\Psi: P \rightarrow \bar{E}$ be defined by

$$
\begin{aligned}
(\Psi m)(s)= & \int_{s}^{s+\widetilde{\omega}} G(s, \theta)\left\{a\left(\psi^{-1}(\theta)\right) H(m(\theta))+\sum_{j=1}^{n} \lambda_{j} \tilde{f}_{j}\left(\psi^{-1}(\theta), v(\theta)\right)\right\} d \theta \\
& +\sum_{i: s_{i} \in[s, s+\tilde{\omega}]} G\left(s, s_{i}\right)\left(\left(\Phi B_{i} \Phi^{-1}\right)\left(m\left(s_{i}\right)\right)+\left(\Phi W_{i} \Phi^{-1}\right)\left(m\left(s_{i}\right)\right)\right),
\end{aligned}
$$

where

$$
G(s, \theta)=\frac{e^{\int_{\theta}^{S} a\left(\psi^{-1}(r)\right) g\left(\left(\Phi^{-1} m\right)(r)\right) \mathrm{d} r}}{1-e^{\int_{\tilde{\omega}}^{0} a\left(\psi^{-1}(r)\right) g\left(\left(\Phi^{-1} m\right)(r)\right) \mathrm{d} r}}, \quad \theta \in[s, s+\tilde{\omega}] .
$$

By the assumptions, we have

$$
\frac{r_{0}^{L}}{1-r_{0}^{L}} \leq G(s, \theta) \leq \frac{1}{1-r_{0}^{l}}
$$

Lemma 2.15. $m$ is an $\tilde{\omega}$-periodic solution of (2.62) if and only if $m$ is a fixed point of the operator $\Psi$.

Proof. If $m(s)$ is an $\widetilde{\omega}$-periodic solution of (2.62), for any $s \in \mathbb{R}$, there exists $i \in \mathbb{Z}$ such that $s_{i}$ is the first impulsive point after $s$. Hence, for $\theta \in\left[s, s_{i}\right]$, we have

$$
\begin{aligned}
m(\theta)= & e^{\int_{s}^{\theta} a\left(\psi^{-1}(\tau)\right) g\left(\left(\Phi^{-1} m\right)(\tau)\right) \mathrm{d} \tau} \\
& -\int_{s}^{\theta} e^{\int_{r}^{\theta} a\left(\psi^{-1}(\tau)\right) g\left(\left(\Phi^{-1} m\right)(\tau)\right) \mathrm{d} \tau}\left\{a\left(\psi^{-1}(r)\right) H(m(r))+\sum_{j=1}^{n} \lambda_{j} \tilde{f}_{j}\left(\psi^{-1}(r), v(r)\right)\right\} \mathrm{d} r,
\end{aligned}
$$


then

$$
\begin{aligned}
m\left(s_{i}\right)= & e^{\int_{s}^{s_{i}} a\left(\psi^{-1}(\tau)\right) g\left(\left(\Phi^{-1} m\right)(\tau)\right) \mathrm{d} \tau} m(s) \\
& -\int_{s}^{s_{i}} e^{\int_{\mathrm{r}}^{s_{i}} a\left(\psi^{-1}(\tau)\right) g\left(\left(\Phi^{-1} m\right)(\tau)\right) \mathrm{d} \tau}\left\{a\left(\psi^{-1}(r)\right) H(m(r))+\sum_{j=1}^{n} \lambda_{j} \tilde{f}_{j}\left(\psi^{-1}(r), v(r)\right)\right\} \mathrm{d} r .
\end{aligned}
$$

Again, for $\theta \in\left(s_{i}, s_{i+1}\right]$, then

$$
\begin{aligned}
& m(s)=e^{\int_{s_{i}}^{\theta} a\left(\psi^{-1}(\tau)\right) g\left(\left(\Phi^{-1} m\right)(\tau)\right) \mathrm{d} \tau} m\left(s_{i}^{+}\right) \\
& -\int_{S_{i}}^{\theta} e^{\int_{r}^{\theta} a\left(\psi^{-1}(\tau)\right) g\left(\left(\Phi^{-1} m\right)(\tau)\right) \mathrm{d} \tau}\left\{a\left(\psi^{-1}(r)\right) H(m(r))+\sum_{j=1}^{n} \lambda_{j} \tilde{f}_{j}\left(\psi^{-1}(r), \nu(r)\right)\right\} \mathrm{d} r \\
& =e^{\int_{s_{i}}^{\theta} a\left(\psi^{-1}(\tau)\right) g\left(\left(\Phi^{-1} m\right)(\tau)\right) \mathrm{d} \tau}\left\{\left(\Phi B_{i} \Phi^{-1}\right)\left(m\left(s_{i}\right)\right)+\left(\Phi W_{i} \Phi^{-1}\right)\left(m\left(s_{i}\right)\right)+m\left(s_{i}\right)\right\} \\
& -\int_{S_{i}}^{\theta} e^{\int_{r}^{\theta} a\left(\psi^{-1}(\tau)\right) g\left(\left(\Phi^{-1} m\right)(\tau)\right) \mathrm{d} \tau}\left\{a\left(\psi^{-1}(r)\right) H(m(r))+\sum_{j=1}^{n} \lambda_{j} \tilde{f}_{j}\left(\psi^{-1}(r), v(r)\right)\right\} \mathrm{d} r \\
& =e^{\int_{s_{i}}^{\theta} a\left(\psi^{-1}(\tau)\right) g\left(\left(\Phi^{-1} m\right)(\tau)\right) \mathrm{d} \tau}\left(\left(\Phi B_{i} \Phi^{-1}\right)\left(m\left(s_{i}\right)\right)+\left(\Phi W_{i} \Phi^{-1}\right)\left(m\left(s_{i}\right)\right)\right) \\
& -\int_{S_{i}}^{\theta} e^{\int_{r}^{\theta} a\left(\psi^{-1}(\tau)\right) g\left(\left(\Phi^{-1} m\right)(\tau)\right) \mathrm{d} \tau}\left\{a\left(\psi^{-1}(r)\right) H(m(r))+\sum_{j=1}^{n} \lambda_{j} \tilde{f}_{j}\left(\psi^{-1}(r), v(r)\right)\right\} \mathrm{d} r \\
& +e^{\int_{s_{i}}^{\theta} a\left(\psi^{-1}(\tau)\right) g\left(\left(\Phi^{-1} m\right)(\tau)\right) \mathrm{d} \tau} m\left(s_{i}\right), \\
& e^{\int_{s_{i}}^{\theta} a\left(\psi^{-1}(\tau)\right) g\left(\left(\Phi^{-1} m\right)(\tau)\right) \mathrm{d} \tau} m\left(s_{i}\right) \\
& =e^{\int_{s_{i}}^{\theta} a\left(\psi^{-1}(\tau)\right) g\left(\left(\Phi^{-1} m\right)(\tau)\right) \mathrm{d} \tau}\left\{e^{\int_{s}^{s_{i}} a\left(\psi^{-1}(\tau)\right) g\left(\left(\Phi^{-1} m\right)(\tau)\right) \mathrm{d} \tau} m(s)\right. \\
& -\int_{S}^{S_{i}} e^{\int_{r}^{S_{i}} a\left(\psi^{-1}(\tau)\right) g\left(\left(\Phi^{-1} m\right)(\tau)\right) \mathrm{d} \tau} \\
& \left.\times\left\{a\left(\psi^{-1}(r)\right) H(m(r))+\sum_{j=1}^{n} \lambda_{j} \tilde{f}_{j}\left(\psi^{-1}(r), v(r)\right)\right\} \mathrm{d} r\right\}
\end{aligned}
$$


Journal of Applied Mathematics

$$
\begin{aligned}
= & e^{\int_{s}^{\theta} a\left(\psi^{-1}(\tau)\right) g\left(\left(\Phi^{-1} m\right)(\tau)\right) \mathrm{d} \tau} m(s) \\
& -\int_{s}^{s_{i}} e^{\int_{r}^{\theta} a\left(\psi^{-1}(\tau)\right) g\left(\left(\Phi^{-1} m\right)(\tau)\right) \mathrm{d} \tau}\left\{a\left(\psi^{-1}(r)\right) H(m(r))+\sum_{j=1}^{n} \lambda_{j} \tilde{f}_{j}\left(\psi^{-1}(r), \nu(r)\right)\right\} \mathrm{d} r .
\end{aligned}
$$

So we can obtain

$$
\begin{aligned}
m(s)= & e^{\int_{s}^{\theta} a\left(\psi^{-1}(\tau)\right) g\left(\left(\Phi^{-1} m\right)(\tau)\right) \mathrm{d} \tau} m(s) \\
& -\int_{s}^{\theta} e^{\int_{r}^{\theta} a\left(\psi^{-1}(\tau)\right) g\left(\left(\Phi^{-1} m\right)(\tau)\right) \mathrm{d} \tau}\left\{a\left(\psi^{-1}(r)\right) H(m(r))+\sum_{j=1}^{n} \lambda_{j} \tilde{f}_{j}\left(\psi^{-1}(r), v(r)\right)\right\} \mathrm{d} r \\
& +e^{\int_{s_{i}}^{\theta} a\left(\psi^{-1}(\tau)\right) g\left(\left(\Phi^{-1} m\right)(\tau)\right) \mathrm{d} \tau}\left[\left(\Phi B_{i} \Phi^{-1}\right)\left(m\left(s_{i}\right)\right)+\left(\Phi W_{i} \Phi^{-1}\right)\left(m\left(s_{i}\right)\right)\right] .
\end{aligned}
$$

Repeating the above process for $\theta \in[s, s+\tilde{\omega}]$, we obtain

$$
\begin{aligned}
m(s)= & e^{\int_{s}^{\theta} a\left(\psi^{-1}(\tau)\right) g\left(\left(\Phi^{-1} m\right)(\tau)\right) \mathrm{d} \tau} m(s) \\
& -\int_{s}^{\theta} e^{\int_{r}^{\theta} a\left(\psi^{-1}(\tau)\right) g\left(\left(\Phi^{-1} m\right)(\tau)\right) \mathrm{d} \tau}\left\{a\left(\psi^{-1}(r)\right) H(m(r))+\sum_{j=1}^{n} \lambda_{j} \tilde{f}_{j}\left(\psi^{-1}(r), v(r)\right)\right\} \mathrm{d} r \\
& +\sum_{i: s_{i} \in[s, \theta)} e^{\int_{s_{i}}^{\theta} a\left(\psi^{-1}(\tau)\right) g\left(\left(\Phi^{-1} m\right)(\tau)\right) \mathrm{d} \tau}\left[\left(\Phi^{-1} B_{i} \Phi^{-1}\right)\left(m\left(s_{i}\right)\right)+\left(\Phi W_{i} \Phi^{-1}\right)\left(m\left(s_{i}\right)\right)\right] .
\end{aligned}
$$

Noticing that $m(s)=m(s+\tilde{\omega})$ and $e^{\int_{s+\tilde{\omega}}^{s} a\left(\psi^{-1}(\tau)\right) g\left(\left(\Phi^{-1} m\right)(\tau)\right) \mathrm{d} \tau}=e^{\int_{\tilde{\omega}}^{0} a\left(\psi^{-1}(\tau)\right) g\left(\left(\Phi^{-1} m\right)(\tau)\right) \mathrm{d} \tau}$, we find that $m$ is a fixed point of $\Psi$.

Let $m$ be a fixed point of $\Psi$. If $s \neq s_{i}, i \in \mathbb{Z}$, we have

$$
\begin{aligned}
m^{\prime}(s)= & G(s, s+\tilde{\omega})\left\{a\left(\psi^{-1}(s+\tilde{\omega})\right) H(m(s+\tilde{\omega}))+\sum_{j=1}^{n} \lambda_{j} \tilde{f}_{j}\left(\psi^{-1}(s+\tilde{\omega}), v(s+\tilde{\omega})\right)\right\} \\
& -G(s, s)\left\{a\left(\psi^{-1}(s)\right) H(m(s))+\sum_{j=1}^{n} \lambda_{j} \tilde{f}_{j}\left(\psi^{-1}(s), v(s)\right)\right\} \\
& +a\left(\psi^{-1}(s)\right) g\left(\left(\Phi^{-1} m\right)(s)\right) m(s) .
\end{aligned}
$$

By Lemmas 2.11 and 2.12, we have $\psi(t+\omega)=\psi(t)+\psi(\omega)=\psi(t)+\tilde{\omega}$, so it is easy to have $t+\omega=\psi^{-1}(\psi(t)+\tilde{\omega})=\psi^{-1}(s+\tilde{\omega})$ and $\mathcal{v}(s+\tilde{\omega})=v(s)$. Therefore, we can obtain

$$
m^{\prime}(s)=a\left(\psi^{-1}(s)\right) g\left(\left(\Phi^{-1} m\right)(s)\right) m(s)-\left\{a\left(\psi^{-1}(s)\right) H(m(s))+\sum_{j=1}^{n} \lambda_{j} \tilde{f}_{j}\left(\psi^{-1}(s), v(s)\right)\right\} .
$$


If $s=s_{i}, i \in \mathbb{Z}$, we can get

$$
\begin{aligned}
m\left(s_{i}^{+}\right)-m\left(s_{i}^{-}\right)= & \sum_{j: s_{j} \in\left[s_{i}^{+}, s_{i}^{+}+\tilde{\omega}\right)} G\left(s_{i}, s_{j}\right)\left(\left(\Phi B_{i} \Phi^{-1}\right)\left(m\left(s_{j}\right)\right)+\left(\Phi W_{i} \Phi^{-1}\right)\left(m\left(s_{j}\right)\right)\right) \\
& -\sum_{j: s_{j} \in\left[s_{i}^{-}, s_{i}^{-}+\tilde{\omega}\right)} G\left(s_{i}, s_{j}\right)\left(\left(\Phi B_{i} \Phi^{-1}\right)\left(m\left(s_{j}\right)\right)+\left(\Phi W_{i} \Phi^{-1}\right)\left(m\left(s_{j}\right)\right)\right) \\
= & G\left(s_{i}, s_{i}+\tilde{\omega}\right)\left(\left(\Phi B_{i} \Phi^{-1}\right)\left(m\left(s_{j}\right)+\tilde{\omega}\right)+\left(\Phi W_{i} \Phi^{-1}\right)\left(m\left(s_{j}\right)+\tilde{\omega}\right)\right) \\
& -G\left(s_{i}, s_{i}\right)\left(\left(\Phi B_{i} \Phi^{-1}\right)\left(m\left(s_{j}\right)\right)+\left(\Phi W_{i} \Phi^{-1}\right)\left(m\left(s_{j}\right)\right)\right) \\
= & -\left(\left(\Phi B_{i} \Phi^{-1}\right)\left(m\left(s_{j}\right)\right)+\left(\Phi W_{i} \Phi^{-1}\right)\left(m\left(s_{j}\right)\right)\right) .
\end{aligned}
$$

Therefore, $m$ is $\tilde{w}$-periodic solution of (2.62). The proof is complete.

Lemma 2.16. Assume that $\left(H_{1}\right)-\left(H_{5}\right)$ hold, then $\Psi(P) \subset P$, and $\Psi: P \rightarrow P$ is compact and continuous.

Proof. By the definition of $P$, for $m \in P$, we have

$$
\begin{aligned}
(\Psi m)(s+\tilde{\omega})= & \int_{s+\tilde{\omega}}^{s+2 \tilde{\omega}} G(s+\tilde{\omega}, \theta)\left\{a\left(\psi^{-1}(\theta)\right) H(m(\theta))+\sum_{j=1}^{n} \lambda_{j} \tilde{f}_{j}\left(\psi^{-1}(\theta), v(\theta)\right)\right\} \mathrm{d} \theta \\
& +\sum_{i: s_{i} \in[s+\tilde{\omega}, s+2 \tilde{\omega}]} G\left(s+\tilde{\omega}, s_{i}\right)\left(\left(\Phi B_{i} \Phi^{-1}\right)\left(m\left(s_{i}\right)\right)+\left(\Phi W_{i} \Phi^{-1}\right)\left(m\left(s_{i}\right)\right)\right) \\
= & \int_{s}^{s+\tilde{\omega}} G(s+\tilde{\omega}, \theta+\tilde{\omega})\left\{a\left(\psi^{-1}(\theta+\tilde{\omega})\right) H(m(\theta+\tilde{\omega}))\right. \\
& +\sum_{i: s_{i} \in[s, s+\tilde{\omega}]} G\left(s+\tilde{\omega}, s_{i}\right)\left(\left(\Phi B_{i} \Phi^{-1}\right)\left(m\left(s_{i}\right)\right)+\left(\Phi W_{i} \Phi^{-1}\right)\left(m\left(s_{i}\right)\right)\right) \\
= & \int_{s}^{s+\tilde{\omega}} G(s, \theta)\left\{a\left(\tilde{f}_{j}\left(\psi^{-1}(\theta+\tilde{\omega}), v(\theta+\tilde{\omega})\right)\right\} \mathrm{d} \theta\right. \\
& +\sum_{i: s_{i} \in[s, s+\tilde{\omega}]} G\left(s, s_{i}\right)\left(\left(\Phi B_{i} \Phi^{-1}\right)\left(m\left(s_{i}\right)\right)+\left(\Phi W_{i} \Phi^{-1}\right)\left(m\left(s_{i}\right)\right)\right) \\
= & (\Psi m)(s) .
\end{aligned}
$$


Thus, $(\Psi m)(s+\tilde{\omega})=(\Psi m)(s), s \in \mathbb{R}$. So in view of (2.66), (2.68), for $m \in P, s \in[0, \tilde{\omega}]$, we have

$$
\begin{aligned}
(\Psi m)(s)= & \int_{s}^{s+\tilde{\omega}} G(s, \theta)\left\{a\left(\psi^{-1}(\theta)\right) H(m(\theta))+\sum_{j=1}^{n} \lambda_{j} \tilde{f}_{j}\left(\psi^{-1}(\theta), v(\theta)\right)\right\} \mathrm{d} \theta \\
& +\sum_{i: s_{i} \in[s, s+\tilde{\omega}]} G\left(s, s_{i}\right)\left(\left(\Phi B_{i} \Phi^{-1}\right)\left(m\left(s_{i}\right)\right)+\left(\Phi W_{i} \Phi^{-1}\right)\left(m\left(s_{i}\right)\right)\right) \\
\geq & \frac{r_{0}^{L}}{1-r_{0}^{L}}\left\{\int_{s}^{s+\tilde{\omega}}\left\{a\left(\psi^{-1}(\theta)\right) H(m(\theta))+\sum_{j=1}^{n} \lambda_{j} \tilde{f}_{j}\left(\psi^{-1}(\theta), v(\theta)\right)\right\} \mathrm{d} \theta\right. \\
& \left.+\sum_{i: s_{i} \in[s, s+\tilde{\omega}]}\left(\left(\Phi B_{i} \Phi^{-1}\right)\left(m\left(s_{i}\right)\right)+\left(\Phi W_{i} \Phi^{-1}\right)\left(m\left(s_{i}\right)\right)\right)\right\} \\
\geq & k \frac{1}{1-r_{0}^{l}}\left\{\int_{s}^{s+\tilde{\omega}}\left\{a\left(\psi^{-1}(\theta)\right) H(m(\theta))+\sum_{j=1}^{n} \lambda_{j} \tilde{f}_{j}\left(\psi^{-1}(\theta), v(\theta)\right)\right\} \mathrm{d} \theta\right. \\
& \left.\quad+\sum_{i: s_{i} \in[s, s+\tilde{\omega}]}\left(\left(\Phi B_{i} \Phi^{-1}\right)\left(m\left(s_{i}\right)\right)+\left(\Phi W_{i} \Phi^{-1}\right)\left(m\left(s_{i}\right)\right)\right)\right\} \\
\geq & k\|\Psi m\| . \quad
\end{aligned}
$$

Therefore, $\Psi m \subset P$. Next, we will show that $\Psi$ is continuous and compact. Firstly, we will consider the continuity of $\Psi$. Let $m_{n} \in P$ and $\left\|m_{n}-m\right\| \rightarrow 0$ as $n \rightarrow+\infty$, then $m \in P$ and $\left|m_{n}(s)-m(s)\right| \rightarrow 0$ as $n \rightarrow+\infty$ for any $s \in[0, \tilde{\omega}]$. By the continuity of $f_{j}(j=1,2, \ldots, n), g, \Phi, \Phi^{-1}, W_{i}(i=1,2, \ldots, p)$, for any $s \in[0, \tilde{\omega}]$ and $\varepsilon>0$, we have

$$
\left|H\left(m_{n}(s)\right)-H(m(s))\right| \leq \frac{1-r_{0}^{l}}{3 \tilde{w} \bar{a}} \varepsilon
$$

and denote $v_{n}(s):=\left(\Phi^{-1} m_{n}\right)\left(\psi\left(\psi^{-1}(s)-v_{j}\left(\psi^{-1}(s)\right)\right)\right)$, and it is easy to see that $\left\|m_{n}-m\right\| \rightarrow 0$ as $n \rightarrow+\infty$ implies $\left\|v_{n}-v\right\| \rightarrow 0$ as $n \rightarrow+\infty$, thus

$$
\begin{aligned}
\left|\tilde{f}_{j}\left(\psi^{-1}(s), v_{n}(s)\right)-\tilde{f}_{j}\left(\psi^{-1}(s), v(s)\right)\right| \leq \frac{1-r_{0}^{l}}{3 \tilde{\omega} \lambda_{j} n} \varepsilon, \quad j=1,2, \ldots, n, \\
\mid\left(\left(\Phi B_{i} \Phi^{-1}\right)\left(m_{n}\left(s_{i}\right)\right)+\left(\Phi W_{i} \Phi^{-1}\right)\left(m_{n}\left(s_{i}\right)\right)\right) \\
-\left(\left(\Phi B_{i} \Phi^{-1}\right)\left(m\left(s_{i}\right)\right)+\left(\Phi W_{i} \Phi^{-1}\right)\left(m\left(s_{i}\right)\right)\right) \mid \leq \frac{1-r_{0}^{l}}{3 \tilde{\omega} p} \varepsilon
\end{aligned}
$$


where $n$ is sufficiently large. For $s \in[0, \tilde{\omega}]$, we have

$$
\begin{aligned}
&\left\|\Psi m_{n}-\Psi m\right\|=\sup _{s \in[0, \tilde{\omega}]}\{\mid \mid \int_{s}^{s+\tilde{\omega}} G(s, \theta)\left\{a\left(\psi^{-1}(\theta)\right) H\left(m_{n}(\theta)\right)+\sum_{j=1}^{n} \lambda_{j} \tilde{f}_{j}\left(\psi^{-1}(\theta), v_{n}(\theta)\right)\right\} \mathrm{d} \theta \\
&+\sum_{i: s_{i} \in[s, s+\tilde{\omega}]} G\left(s, s_{i}\right)\left(\left(\Phi B_{i} \Phi^{-1}\right)\left(m_{n}\left(s_{i}\right)\right)+\left(\Phi W_{i} \Phi^{-1}\right)\left(m_{n}\left(s_{i}\right)\right)\right) \\
&-\int_{s}^{s+\tilde{\omega}} G(s, \theta)\left\{a\left(\psi^{-1}(\theta)\right) H(m(\theta))+\sum_{j=1}^{n} \lambda_{j} \tilde{f}_{j}\left(\psi^{-1}(\theta), v(\theta)\right)\right\} \mathrm{d} \theta \\
&\left.-\sum_{i: s_{i} \in[s, s+\tilde{\omega}]} G\left(s, s_{i}\right)\left(\left(\Phi B_{i} \Phi^{-1}\right)\left(m\left(s_{i}\right)\right)+\left(\Phi W_{i} \Phi^{-1}\right)\left(m\left(s_{i}\right)\right)\right) \mid\right\} \\
&=\sup _{s \in[0, \tilde{\omega}]}\left\{\mid \int_{s}^{s+\tilde{\omega}} G(s, \theta)\left\{a\left(\psi^{-1}(s)\right)\left(H\left(m_{n}(s)\right)-H(m(s))\right)\right.\right. \\
&+\sum_{i: s_{i} \in[s, s+\tilde{\omega})} G\left(s, s_{i}\right)\left(\left(\left(\Phi B_{i} \Phi^{-1}\right)\left(m_{n}\left(s_{i}\right)\right)+\left(\Phi W_{i} \Phi^{-1}\right)\left(m_{n}\left(s_{i}\right)\right)\right)\right. \\
&\left.\left.-\left(\left(\Phi B_{i} \Phi^{-1}\right)\left(m\left(s_{i}\right)\right)+\left(\Phi W_{i} \Phi^{-1}\right)\left(m\left(s_{i}\right)\right)\right)\right) \mid\right\} \\
& \leq \frac{\tilde{\omega}}{1-r_{0}^{l}}\left\{\begin{array}{l}
n \\
\left.\bar{a} \frac{1-r_{0}^{l}}{3 \tilde{\omega} \bar{a}} \varepsilon+\sum_{j=1}^{n} \lambda_{j} \frac{1-r_{0}^{l}}{3 \tilde{\omega} \lambda_{j} n} \varepsilon+p \frac{1-r_{0}^{l}}{3 \tilde{\omega} p} \varepsilon\right\}=\varepsilon .
\end{array}\right.
\end{aligned}
$$

Therefore, $\Psi$ is continuous on $P$.

Next, we prove that $\Psi$ is a compact operator. Let $S \subset P$ be an arbitrary bounded set in $P$, then there exists a number $L_{0}>0$ such that $\|m\|<L_{0}$ for any $m \in S$. We prove that $\overline{\Psi S}$ is compact. In fact, from $\left(\mathrm{H}_{1}\right)$, one has $W_{i}(0)=0, i=1,2, \ldots$; by (2.53), it is easy to see that for any $z \in \mathbb{R}$, one has the following:

$$
\left\|W_{i}(z)\right\| \leq k\left(l_{0}\right) l_{0}\|z\|+\left\|W_{i}(0)\right\|=k\left(l_{0}\right) l_{0}\|z\|, \quad i=1,2, \ldots
$$

So for any $\left\{m_{n}\right\}_{n \in \mathbb{N}} \subset S$ and $s \in[0, \tilde{\omega}]$, we have

$$
\begin{aligned}
\left\|\Psi m_{n}\right\|=\sup _{s \in[0, \tilde{\omega}]} & \left\{\mid \int_{s}^{s+\tilde{\omega}}(s, \theta)\left\{a\left(\psi^{-1}(\theta)\right) H\left(m_{n}(\theta)\right)+\sum_{j=1}^{n} \lambda_{j} \tilde{f}_{j}\left(\psi^{-1}(\theta), v_{n}(\theta)\right)\right\} \mathrm{d} \theta\right. \\
& \left.+\sum_{i: s_{i} \in[s, s+\tilde{\omega}]} G\left(s, s_{i}\right)\left(\left(\Phi B_{i} \Phi^{-1}\right)\left(m_{n}\left(s_{i}\right)\right)+\left(\Phi W_{i} \Phi^{-1}\right)\left(m_{n}\left(s_{i}\right)\right)\right) \mid\right\}
\end{aligned}
$$


Journal of Applied Mathematics

$$
\begin{aligned}
\leq & \frac{\tilde{\omega}}{1-r_{0}^{l}}\left\{\bar{a} \frac{L \bar{c}}{1-\bar{c}}+\sum_{j=1}^{n} \max _{v \in\left[-L_{0}^{\prime}, L_{0}^{\prime}\right] s \in[0, \tilde{\omega}]} \lambda_{j} \tilde{f}_{j}\left(\psi^{-1}(s), v(s)\right)\right. \\
& \left.+L_{0} \sum_{i=1}^{p}\left(B_{i}+k\left(l_{0}\right) l_{0}\right)\right\}:=K, \\
\left\|\left(\Psi m_{n}\right)^{\prime}\right\|=\sup _{s \in[0, \tilde{\omega}]}\left\{\mid a\left(\psi^{-1}(s)\right) g\left(\left(\Phi^{-1} m_{n}\right)(s)\right) m_{n}(s)\right. & \\
& \left.-\left\{a\left(\psi^{-1}(s)\right) H\left(m_{n}(s)\right)+\sum_{j=1}^{n} \lambda_{j} \tilde{f}_{j}\left(\psi^{-1}(s), v_{n}(s)\right)\right\} \mid\right\} \\
\leq & \bar{a} L_{0}\left\|\Psi m_{n}\right\|+\bar{a} \frac{L \bar{c}}{1-\bar{c}}+\sum_{j=1}^{n} \max _{v \in\left[-L_{0}^{\prime}, L_{0}^{\prime}\right] s \in[0, \tilde{\omega}]} \lambda_{j} \tilde{f}_{j}\left(\psi^{-1}(s), v(s)\right) \\
\leq & \bar{a} L_{0} K+\bar{a} \frac{L \bar{c}}{1-\bar{c}}+\sum_{j=1}^{n} \max _{v \in\left[-L_{0}^{\prime}, L_{0}^{\prime}\right] s \in[0, \tilde{\omega}]} \lambda_{j} \tilde{f}_{j}\left(\psi^{-1}(s), v(s)\right):=Q,
\end{aligned}
$$

where $L_{0}^{\prime}=L_{0} /(1-\bar{c})$, which implies that $\left\{\Psi m_{n}\right\}_{n \in \mathbb{N}}$ and $\left\{\left(\Psi m_{n}\right)^{\prime}\right\}_{n \in \mathbb{N}}$ are uniformly bounded on $[0, \tilde{\omega}]$. Therefore, there exists a subsequence of $\left\{\Psi m_{n}\right\}_{n \in \mathbb{N}}$ which converges uniformly on $[0, \tilde{\omega}]$; namely, $\overline{\Psi S}$ is compact. The proof is complete.

\section{Main Results}

Our main results of this paper are as follows.

Theorem 3.1. Assume that $\left(H_{1}\right)-\left(H_{5}\right)$ hold, $0 \leq c(t)<1$, for a sufficiently small Lipschitz constant $l_{0}$; suppose that the following conditions hold:

$\left(\mathrm{H}_{6}\right) \tilde{\alpha}_{0}=(1-\bar{c})\left(1-r_{0}^{l}\right)-\tilde{\omega} \bar{a} L \bar{c}-(1-\bar{c}) \sum_{i=1}^{p}\left(B_{i}+k\left(l_{0}\right) l_{0}\right)>0$.

$\left(\mathrm{H}_{7}\right)$ There exist positive constants $\beta_{1}, \beta_{2}$, and $\beta_{4}$ with $0<\beta_{1}<\beta_{2}<\beta_{4}$ such that

$$
\frac{\sup _{s \in[0, \tilde{\omega}]} f_{j}\left(\psi^{-1}(s), \beta_{1} /(1-\bar{c})\right)}{\left(\beta_{1} /(1-\bar{c})\right) \tilde{\alpha}_{0}}<\frac{\sup _{s \in[0, \tilde{\omega}]} f_{j}\left(\psi^{-1}(s), \beta_{4} /(1-\bar{c})\right)}{\left(\beta_{4} /(1-\bar{c})\right) \tilde{\alpha}_{0}}<\frac{\inf _{s \in[0, \tilde{\omega}]} f_{j}\left(\psi^{-1}(s), \tilde{\alpha} \beta_{2}\right)}{\tilde{\alpha} \beta_{2} \tilde{\beta}_{0}}
$$

where $\widetilde{\beta}_{0}=\left(\left(1-r_{0}^{L}\right) / \tilde{\alpha} r_{0}^{L}\right)-\tilde{\omega} \underline{a} \underline{\underline{c}}$ and $\tilde{\omega}=\psi(\omega)$.

Then for all $j=1,2, \ldots, n, \lambda_{j} \in\left(\lambda_{j_{1}}, \lambda_{j_{2}}\right],(1.2)$ has at least three positive $\omega$-periodic solutions, where

$$
\lambda_{j_{1}}=\frac{\tilde{\alpha} \beta_{2} \tilde{\beta}_{0}}{\tilde{\omega} n \inf _{s \in[0, \tilde{\omega}]} f_{j}\left(t, \tilde{\alpha} \beta_{2}\right)}, \quad \lambda_{j_{2}}=\frac{\left(\beta_{4} /(1-\bar{c})\right) \tilde{\alpha}_{0}}{\tilde{\omega} n \sup _{s \in[0, \tilde{\omega}]} f_{j}\left(t, \beta_{4} /(1-\bar{c})\right)}, \quad j=1,2, \ldots, n .
$$


Proof. First of all, since $0<\tilde{\alpha}<1 /(1-\bar{c})$ and $0<r_{0}<1$, we have $\tilde{\alpha}_{0}>0$, so

$$
\begin{aligned}
\tilde{\beta}_{0}= & \frac{1-r_{0}^{L}}{\tilde{\alpha} r_{0}^{L}}-\tilde{\omega} \underline{a} \underline{\underline{c}}>>\frac{1-r_{0}^{L}}{\tilde{\alpha} r_{0}^{L}}-\tilde{\omega} \bar{a} l \bar{c}>\frac{1-r_{0}^{L}}{\tilde{\alpha}}-(1-\bar{c})\left(1-r_{0}^{l}\right) \\
& +(1-\bar{c}) \sum_{i=1}^{p}\left(B_{i}+k\left(l_{0}\right) l_{0}\right) \\
> & (1-\bar{c})\left(r_{0}^{l}-r_{0}^{L}\right)+(1-\bar{c}) \sum_{i=1}^{p}\left(B_{i}+k\left(l_{0}\right) l_{0}\right)>0 .
\end{aligned}
$$

Furthermore, $0<\lambda_{j_{1}}<\lambda_{j_{2}}$ in view of (3.1).

Now, define for each $\lambda_{j} \in\left(\lambda_{j_{1}}, \lambda_{j_{2}}\right]$ and $m \in P$ a mapping $\Psi: P \rightarrow P$ by

$$
\begin{aligned}
(\Psi m)(s)= & \int_{s}^{s+\tilde{\omega}} G(s, \theta)\left\{a\left(\psi^{-1}(\theta)\right) H(m(\theta))+\sum_{j=1}^{n} \lambda_{j} \tilde{f}_{j}\left(\psi^{-1}(\theta), v(\theta)\right)\right\} \mathrm{d} \theta \\
& +\sum_{i: s_{i} \in[s, s+\tilde{\omega}]} G\left(s, s_{\mathrm{i}}\right)\left(\left(\Phi B_{i} \Phi^{-1}\right)\left(m\left(s_{i}\right)\right)+\left(\Phi W_{i} \Phi^{-1}\right)\left(m\left(s_{i}\right)\right)\right),
\end{aligned}
$$

and a function $\rho: P \rightarrow[0, \infty)$ by

$$
\rho(m)=\min _{s \in[0, \tilde{\omega}]} m(s)
$$

For $m \in \overline{P_{\beta_{4}}}$, by Lemma 2.13 , we have

$$
0<\left(\Phi^{-1} m\right)(s)<\frac{\beta_{4}}{1-\bar{c}}
$$

It follows from (2.51), (2.68), (3.6), and $\left(\mathrm{H}_{2}\right)$, for all $j=1,2, \ldots, n, \lambda_{j} \in\left(\lambda_{j 1}, \lambda_{j 2}\right]$ and $m \in \overline{P_{\beta_{4}}}$ that

$$
\begin{aligned}
(\Psi m)(s)= & \int_{s}^{s+\tilde{\omega}} G(s, \theta)\left\{a\left(\psi^{-1}(\theta)\right) H(m(\theta))+\sum_{j=1}^{n} \lambda_{j} \tilde{f}_{j}\left(\psi^{-1}(\theta), v(\theta)\right)\right\} \mathrm{d} \theta \\
& +\sum_{i: s_{i} \in[s, s+\tilde{\omega}]} G\left(s, s_{i}\right)\left(\left(\Phi B_{i} \Phi^{-1}\right)\left(m\left(s_{i}\right)\right)+\left(\Phi W_{i} \Phi^{-1}\right)\left(m\left(s_{i}\right)\right)\right) \\
\leq & \frac{1}{1-r_{0}^{l}}\left\{\int_{s}^{s+\tilde{\omega}}\left\{a\left(\psi^{-1}(\theta)\right) H(m(\theta))+\sum_{j=1}^{n} \lambda_{j} \tilde{f}_{j}\left(\psi^{-1}(\theta), v(\theta)\right)\right\} \mathrm{d} \theta\right. \\
& \left.+\sum_{i: s_{i} \in[s, s+\tilde{\omega}]}\left(\left(\Phi B_{i} \Phi^{-1}\right)\left(m\left(s_{i}\right)\right)+\left(\Phi W_{i} \Phi^{-1}\right)\left(m\left(s_{i}\right)\right)\right)\right\} \\
\leq & \frac{1}{1-r_{0}^{l}}\left\{\bar{a} \tilde{\omega} \frac{L \bar{c}}{1-\bar{c}} \beta_{4}+\tilde{\omega} \sum_{j=1}^{n} \lambda_{j 2} \sup _{s \in[0, \tilde{\omega}]} \tilde{f}_{j}\left(\psi^{-1}(s), \frac{\beta_{4}}{1-\bar{c}}\right)+\beta_{4} \sum_{i=1}^{p}\left(b_{i}+k\left(l_{0}\right) l_{0}\right)\right\}
\end{aligned}
$$


Journal of Applied Mathematics

$$
\begin{aligned}
&=\frac{1}{1-r_{0}^{l}}\left\{\bar{a} \tilde{\omega} \frac{L \bar{c}}{1-\bar{c}} \beta_{4}+\sum_{j=1}^{n} \frac{\left(\beta_{4} /(1-\bar{c})\right) \alpha_{0}}{\tilde{\omega} n \sup _{s \in[0, \tilde{\omega}]} \tilde{f}_{j}\left(\psi^{-1}(s), \beta_{4} /(1-\bar{c})\right)} \sup _{s \in[0, \tilde{\omega}]} \tilde{f}_{j}\left(\psi^{-1}(s), \frac{\beta_{4}}{1-\bar{c}}\right)\right. \\
&\left.+\beta_{4} \sum_{i=1}^{p}\left(B_{i}+k\left(l_{0}\right) l_{0}\right)\right\} \leq \beta_{4} .
\end{aligned}
$$

By Lemma 2.16, we know that $\Psi$ is completely continuous on $\overline{P_{\beta_{4}}}$.

We now assert that the condition (2) of Lemma 2.1 holds. Indeed, if $m \in \overline{P_{\beta_{1}}}$, then similar to above argument, by (3.1), we have

$$
\begin{aligned}
& (\Psi m)(s)=\int_{s}^{s+\tilde{\omega}} G(s, \theta)\left\{a\left(\psi^{-1}(\theta)\right) H(m(\theta))+\sum_{j=1}^{n} \lambda_{j} \tilde{f}_{j}\left(\psi^{-1}(\theta), v(\theta)\right)\right\} \mathrm{d} \theta \\
& +\sum_{i: s_{i} \in[s, s+\tilde{\omega}]} G\left(s, s_{i}\right)\left(\left(\Phi B_{i} \Phi^{-1}\right)\left(m\left(s_{i}\right)\right)+\left(\Phi W_{i} \Phi^{-1}\right)\left(m\left(s_{i}\right)\right)\right) \\
& \leq \frac{1}{1-r_{0}^{l}}\left\{\int_{s}^{s+\tilde{\omega}}\left\{a\left(\psi^{-1}(\theta)\right) H(m(\theta))+\sum_{j=1}^{n} \lambda_{j} \tilde{f}_{j}\left(\psi^{-1}(\theta), v(\theta)\right)\right\} \mathrm{d} \theta\right. \\
& \left.+\sum_{i: s_{i} \in[s, s+\tilde{\omega}]}\left(\left(\Phi B_{i} \Phi^{-1}\right)\left(m\left(s_{i}\right)\right)+\left(\Phi W_{i} \Phi^{-1}\right)\left(m\left(s_{i}\right)\right)\right)\right\} \\
& \leq \frac{1}{1-r_{0}^{l}}\left\{\int_{s}^{s+\tilde{\omega}}\left\{a\left(\psi^{-1}(\theta)\right) H(m(\theta))+\sum_{j=1}^{n} \lambda_{j} \tilde{f}_{j}\left(\psi^{-1}(\theta), v(\theta)\right)\right\} \mathrm{d} \theta\right. \\
& \left.+\sum_{i: s_{i} \in[s, s+\tilde{\omega}]}\left(\left(\Phi B_{i} \Phi^{-1}\right)\left(m\left(s_{i}\right)\right)+\left(\Phi W_{i} \Phi^{-1}\right)\left(m\left(s_{i}\right)\right)\right)\right\} \\
& \leq \frac{1}{1-r_{0}^{l}}\left\{\bar{a} \tilde{\omega} \frac{L \bar{c}}{1-\bar{c}} \beta_{1}+\tilde{\omega} \sum_{j=1}^{n} \lambda_{j 2} \sup _{s \in[0, \tilde{\omega}]} \tilde{f}_{j}\left(\psi^{-1}(s), \frac{\beta_{1}}{1-\bar{c}}\right)+\beta_{1} \sum_{i=1}^{p}\left(B_{i}+k\left(l_{0}\right) l_{0}\right)\right\} \\
& =\frac{1}{1-r_{0}^{l}}\left\{\bar{a} \tilde{\omega} \frac{L \bar{c}}{1-\bar{c}} \beta_{1}+\sum_{j=1}^{n} \frac{\left(\beta_{4} /(1-\bar{c})\right) \alpha_{0}}{\tilde{\omega} n \sup _{s \in[0, \tilde{\omega}]} \tilde{f}_{j}\left(\psi^{-1}(s), \beta_{4} /(1-\bar{c})\right)} \sup _{s \in[0, \tilde{\omega}]} \tilde{f}_{j}\left(\psi^{-1}(s), \frac{\beta_{1}}{1-\bar{c}}\right)\right. \\
& \left.+\beta_{1} \sum_{i=1}^{p}\left(B_{i}+k\left(l_{0}\right) l_{0}\right)\right\} \\
& <\frac{1}{1-r_{0}^{l}}\left\{\bar{a} \tilde{\omega} \frac{L \bar{c}}{1-\bar{c}} \beta_{1}+\frac{\beta_{4}}{1-\bar{c}} \alpha_{0} \frac{\beta_{1}}{\beta_{4}}+\beta_{1} \sum_{i=1}^{p}\left(B_{i}+k\left(l_{0}\right) l_{0}\right)\right\}=\beta_{1} .
\end{aligned}
$$

Hence, $\|\Psi m\|<\beta_{1}$ holds. 
Choose a positive constant $\beta_{3}$ such that $0<\beta_{2}<k \beta_{3}<\beta_{3} \leq \beta_{4}$. Next, we show that the condition (1) of Lemma 2.1 holds. Obviously, $\rho$ is a concave continuous function on $P$ with $\rho(m) \leq\|m\|$ for $m \in \overline{P_{\beta_{4}}}$. We notice that if $m(s)=(2 / 5) \beta_{2}+(3 / 5) \beta_{3}$ for $s \in[0, \tilde{\omega}]$, then $m \in\left\{m \in P\left(\rho, \beta_{2}, \beta_{3}\right): \rho(m)>\beta_{2}\right\}$ which implies $\left\{m \in P\left(\rho, \beta_{2}, \beta_{3}\right): \rho(m)>\beta_{2}\right\} \neq \emptyset$. For $m \in P\left(\rho, \beta_{2}, \beta_{3}\right)$, we have

$$
\beta_{2} \leq \rho(m)=\min _{s \in[0, \tilde{\omega}]} m(s) \leq\|m\| \leq \beta_{3}
$$

which implies, from (2.50), that

$$
\left(\Phi^{-1} m\right)(s) \geq \tilde{\alpha}\|m\| \geq \alpha \beta_{2}
$$

And it is also clear that $\Phi(x)$ is nondecreasing for $x>0$ and $B_{i}, W_{i} \in C\left(\mathbb{R}, \mathbb{R}^{+}\right)$, and we can easily have $\Phi B_{i} \Phi^{-1}, \Phi W_{i} \Phi^{-1} \in C\left(\mathbb{R}, \mathbb{R}^{+}\right)$. Hence

$$
\begin{aligned}
& \rho(\Psi m)=\min _{s \in[0, \tilde{\omega}]}(\Psi m)(s) \\
& =\min _{s \in[0, \tilde{\omega}]}\left\{\int_{s}^{s+\tilde{\omega}} G(s, \theta)\left\{a\left(\psi^{-1}(\theta)\right) H(m(\theta))+\sum_{j=1}^{n} \lambda_{j} \tilde{f}_{j}\left(\psi^{-1}(\theta), \nu(\theta)\right)\right\} \mathrm{d} \theta\right. \\
& \left.+\sum_{i: s_{i} \in[s, s+\tilde{\omega}]} G\left(s, s_{i}\right)\left(\left(\Phi B_{i} \Phi^{-1}\right)\left(m\left(s_{i}\right)\right)+\left(\Phi W_{i} \Phi^{-1}\right)\left(m\left(s_{i}\right)\right)\right)\right\} \\
& \geq \frac{r_{0}^{L}}{1-r_{0}^{L}} \min _{s \in[0, \tilde{\omega}]}\left\{\int_{s}^{s+\tilde{\omega}}\left\{a\left(\psi^{-1}(\theta)\right) H(m(\theta))+\sum_{j=1}^{n} \lambda_{j} \tilde{f}_{j}\left(\psi^{-1}(\theta), \tilde{\alpha} \beta_{2}\right)\right\} \mathrm{d} \theta\right. \\
& \left.+\sum_{i: s_{i} \in[s, s+\tilde{\omega}]}\left(\left(\Phi B_{i} \Phi^{-1}\right)\left(m\left(s_{i}\right)\right)+\left(\Phi W_{i} \Phi^{-1}\right)\left(m\left(s_{i}\right)\right)\right)\right\} \\
& >\frac{r_{0}^{L}}{1-r_{0}^{L}}\left\{\tilde{\omega} \underline{a} \underline{c} \underline{\alpha} \tilde{\alpha} \beta_{2}+\tilde{\omega} \sum_{j=1}^{n} \lambda_{j_{1}} \inf _{s \in[0, \tilde{\omega}]} \tilde{f}_{j}\left(\psi^{-1}(s), \tilde{\alpha} \beta_{2}\right)\right. \\
& \left.+\min _{\beta_{2} \leq m \leq \beta_{3}} \sum_{i=1}^{p}\left(\left(\Phi B_{i} \Phi^{-1}\right)\left(m\left(s_{i}\right)\right)+\left(\Phi W_{i} \Phi^{-1}\right)\left(m\left(s_{i}\right)\right)\right)\right\} \\
& \geq \frac{r_{0}^{L}}{1-r_{0}^{L}}\left\{\tilde{\omega} \underline{a l} \underline{\underline{\alpha}} \beta_{2}+\tilde{\omega} \sum_{j=1}^{n} \frac{\tilde{\alpha} \beta_{2} \tilde{\beta}_{0}}{\tilde{\omega} n \inf _{s \in[0, \tilde{\omega}]} \tilde{f}_{j}\left(\psi^{-1}(s), \tilde{\alpha} \beta_{2}\right)} \inf _{s \in[0, \tilde{\omega}]} \tilde{f}_{j}\left(\psi^{-1}(s), \tilde{\alpha} \beta_{2}\right)\right. \\
& \left.+\min _{\beta_{2} \leq m \leq \beta_{3}} \sum_{i=1}^{p}\left(\left(\Phi B_{i} \Phi^{-1}\right)\left(m\left(s_{i}\right)\right)+\left(\Phi W_{i} \Phi^{-1}\right)\left(m\left(s_{i}\right)\right)\right)\right\}>\beta_{2}
\end{aligned}
$$

for all $m \in P\left(\rho, \beta_{2}, \beta_{3}\right)$. 
Finally, we prove that the condition (3) of Lemma 2.1 holds. Let $m \in P\left(\rho, \beta_{2}, \beta_{4}\right)$ and $\|\Psi m\|>\beta_{3}$, then $\rho(\Psi m)>\beta_{2}$. We notice that (3.4) implies that

$$
\begin{gathered}
\|\Psi m\| \leq \frac{1}{1-r_{0}^{l}}\left\{\int_{s}^{s+\tilde{\omega}}\left\{a\left(\psi^{-1}(\theta)\right) H(m(\theta))+\sum_{j=1}^{n} \lambda_{j} \tilde{f}_{j}\left(\psi^{-1}(\theta), \tilde{\alpha} \beta_{2}\right)\right\} \mathrm{d} \theta\right. \\
\left.+\sum_{i: s_{i} \in[s, s+\tilde{\omega}]}\left(\left(\Phi B_{i} \Phi^{-1}\right)\left(m\left(s_{i}\right)\right)+\left(\Phi W_{i} \Phi^{-1}\right)\left(m\left(s_{i}\right)\right)\right)\right\} .
\end{gathered}
$$

Thus

$$
\begin{aligned}
\rho(\Psi m)= & \min _{s \in[0, \tilde{\omega}]}\left\{\int_{s}^{s+\tilde{\omega}} G(s, \theta)\left\{a\left(\psi^{-1}(\theta)\right) H(m(\theta))+\sum_{j=1}^{n} \lambda_{j} \tilde{f}_{j}\left(\psi^{-1}(\theta), v(\theta)\right)\right\} \mathrm{d} \theta\right. \\
& +\sum_{i: s_{i} \in[s, s+\tilde{\omega}]} G\left(s, s_{i}\right)\left(\left(\Phi B_{i} \Phi^{-1}\right)\left(m\left(s_{i}\right)\right)+\left(\Phi W_{i} \Phi^{-1}\right)\left(m\left(s_{i}\right)\right)\right\} \\
\geq & \frac{r_{0}^{L}}{1-r_{0}^{L}} \min _{s \in[0, \tilde{\omega}]}\left\{\int_{s}^{s+\tilde{\omega}}\left\{a\left(\psi^{-1}(\theta)\right) H(m(\theta))+\sum_{j=1}^{n} \lambda_{j} \tilde{f}_{j}\left(\psi^{-1}(\theta), v(\theta)\right)\right\} \mathrm{d} \theta\right. \\
& \left.+\sum_{i: s_{i} \in[s, s+\tilde{\omega}]}\left(\left(\Phi B_{i} \Phi^{-1}\right)\left(m\left(s_{i}\right)\right)+\left(\Phi W_{i} \Phi^{-1}\right)\left(m\left(s_{i}\right)\right)\right)\right\} \\
\geq & \frac{r_{0}^{L}\left(1-r_{0}^{l}\right)}{1-r_{0}^{l}}\|\Psi m\| \geq k \beta_{3}>\beta_{2} .
\end{aligned}
$$

To sum up, all the hypotheses of Lemma 2.1 are satisfied. Hence $\Psi$ has at least three positive fixed points. That is, (1.2) has at least three positive $\omega$-periodic solutions. This completes the proof.

Corollary 3.2. Suppose $\left(H_{1}\right)-\left(H_{6}\right)$ hold. If

$$
\begin{aligned}
& \lim _{x \rightarrow \infty} \frac{\sup _{s \in[0, \tilde{\omega}]} f_{j}\left(\psi^{-1}(s), x\right)}{x}=0, \\
& \lim _{x \rightarrow 0} \frac{\sup _{s \in[0, \tilde{\omega}]} f_{j}\left(\psi^{-1}(s), x\right)}{x}=0,
\end{aligned}
$$

where $\tilde{\omega}=\psi(\omega)$; then (1.2) has at least three positive $\omega$-periodic solutions. 
Proof. In view of (3.14), we can choose $\beta_{4}>\beta_{2}>0$ such that the second inequality in (3.1) holds, and in view of (3.15), we can choose $\beta_{1} \in\left(0, \beta_{2}\right)$ such that the first inequality in (3.1) holds. Therefore, the conclusion of Theorem 3.1 holds. This completes the proof.

\section{An Example}

Let us consider the variable time scale $\mathbb{T}_{0}(x)$ constructed by $t_{i}=i, \tau_{i}(x)=(-1)^{i} l_{0} \sin x$, where $|x|>1$ for all $t \in \mathbb{T}_{0}(x), 0<l_{0}<(1 / 2)$ and consider $\pi$-periodic system:

$$
\begin{aligned}
\left(x(t)+\frac{1}{9}|\cos t| x(t-\alpha)\right)^{\prime} & =\frac{1}{\pi}|\sin t|\left(\frac{1}{3}+\frac{1}{3} e^{-x}\right) x(t)-\frac{l_{0}}{3 n} \sum_{j=1}^{n} \lambda_{j} x^{1 / 2}(t) \ln \left(x\left(t-e^{(1 / j)|\sin t|}\right)+1\right), \quad(t, x) \in \mathbb{T}_{0}(x), \\
x^{+} & =0.03\left(\frac{2}{3}\right)^{i} x+0.02 l_{0} \sin x+x, \\
t^{+} & =2 i+1-l_{0} \sin x,
\end{aligned}
$$

where $\alpha$ is a constant, $\lambda_{j}, j=1,2, \ldots, n$ are nonnegative parameters. In this case, $c(t)=(1 / 9)|\cos t|, a(t)=1 / \pi, g(x(t))=(1 / 3)+(1 / 3) e^{-x}, B_{i}=0.03(2 / 3)^{i}, J_{i}(x)=$ $0.02 l_{0} \sin x, v_{j}(t)=e^{(1 / j)|\sin t|}$ and $f_{j}\left(t, x\left(t-v_{j}(t)\right)\right)=\left(l_{0} / 3 n\right) x^{1 / 2}(t) \ln \left(x\left(t-v_{j}(t)\right)+1\right), j=$ $1,2, \ldots, n, i \in \mathbb{N}$. Obviously, $\left(\mathrm{H}_{1}\right)-\left(\mathrm{H}_{3}\right)$ are satisfied, and it is easy to see that (3.14) and (3.15) hold.

By the formula of $\psi$-substitution and $\delta_{k}=1$, one can find

$$
\tilde{\omega}=\psi(\omega)=\omega-\sum_{0<2 k<\omega} \delta_{k}=\pi-1
$$

Clearly, $L=(2 / 3), l=(1 / 3), 0 \leq c(t) \leq(1 / 9)<1, r_{0}=e^{-(\pi-1)(1 / \pi)} \approx 0.5058$ and $\bar{c}=1 / 9, \underline{c}=0$, so we can find $\delta=(\underline{c}+\bar{c}) / 2=1 / 18$, and it is easy to check that $((2 \delta-$ $\left.\underline{c})\left(1-\underline{c}^{2}\right)\right) /\left(1-(2 \delta-\underline{c})^{2}\right)=9 / 80 \approx 0.1125<\left(r_{0}^{L}\left(1-r_{0}^{l}\right)\right) /\left(1-r_{0}^{L}\right)=0.3532$. Thus, $\left(\mathrm{H}_{4}\right)$ holds. Furthermore, we also have

$$
\begin{aligned}
\left\|\frac{\partial f_{j}}{\partial x}\right\| & =\frac{l_{0}}{3 n}\left\|\frac{\ln (x+1)}{2 x^{1 / 2}}+\frac{x^{1 / 2}}{x+1}\right\| \leq \frac{l_{0}}{3 n}\left(\left\|\frac{\ln (x+1)^{1 / 2}}{x^{1 / 2}}\right\|+\left\|\frac{x^{1 / 2}}{x+1}\right\|\right) \\
& \leq \frac{l_{0}}{3 n}\left(\left\|\frac{(x+1)^{1 / 2}}{x^{1 / 2}}\right\|+\left\|\frac{x^{1 / 2}}{x+1}\right\|\right)=\frac{l_{0}}{3 n}\left(\left\|\left(1+\frac{1}{x}\right)^{1 / 2}\right\|+\left\|\frac{x^{1 / 2}}{x+1}\right\|\right) \\
& <\frac{l_{0}}{3 n}(2+1)=\frac{l_{0}}{n} .
\end{aligned}
$$

Hence, for any $x_{1}, x_{2} \in \mathbb{R}$, one can get

$$
\sum_{j=1}^{n}\left|f_{j}\left(t, x_{1}\right)-f_{j}\left(t, x_{2}\right)\right| \leq\left|\frac{\partial f_{j}}{\partial x}\right|\left|x_{1}-x_{2}\right|<n \cdot \frac{l_{0}}{n}\left|x_{1}-x_{2}\right|=l_{0}\left|x_{1}-x_{2}\right| .
$$


So $\left(\mathrm{H}_{5}\right)$ is satisfied. For a sufficiently small $l_{0}$, one can also have

$$
\tilde{\alpha}_{0}=(1-\bar{c})\left(1-r_{0}^{l}\right)-\tilde{\omega} \bar{a} L \bar{c}-(1-\bar{c}) \sum_{i=1}^{p}\left(B_{i}+k\left(l_{0}\right) l_{0}\right)=0.0768-\frac{8}{9} \sum_{i=1}^{p} k\left(l_{0}\right) l_{0}
$$

since $k\left(l_{0}\right)$ is a bounded function; for a sufficiently small $l_{0}$, one can have $\tilde{\alpha}_{0}>0$ such that $\left(\mathrm{H}_{6}\right)$ holds. Therefore, according to Corollary 3.2, (4.1) has at least three positive $\pi$-periodic solutions.

\section{Acknowledgment}

This work is supported by the National Natural Sciences Foundation of China under Grant no. 10971183.

\section{References}

[1] M. Bohner and A. Peterson, Dynamic Equations on Time Scales, An Introduction with Applications, Birkhäuser Boston, Boston, Mass, USA, 2001.

[2] V. Lakshmikantham, S. Sivasundaram, and B. Kaymakcalan, Dynamic Systems on Measure Chains, vol. 370 of Mathematics and its Applications, Kluwer Academic, Dodrecht, The Netherlands, 1996.

[3] V. Lakshmikantham and A. S. Vatsala, "Hybrid systems on time scales. Dynamic equations on time scales," Journal of Computational and Applied Mathematics, vol. 141, no. 1-2, pp. 227-235, 2002.

[4] S. Sivasundaram, "Stability of dynamic systems on the time scales," Nonlinear Dynamics and Systems Theory, vol. 2, no. 2, pp. 185-202, 2002.

[5] M. U. Akhmet and M. Turan, "The differential equation on time scales through impulsive differential equations," Nonlinear Analysis, vol. 65, no. 11, pp. 2043-2060, 2006.

[6] M. U. Akhmet and M. Turan, "Differential equations on variable time scales," Nonlinear Analysis, vol. 70, no. 3, pp. 1175-1192, 2009.

[7] M. U. Akhmet, "Perturbations and Hopf bifurcation of the planar discontinuous dynamical system," Nonlinear Analysis, vol. 60, no. 1, pp. 163-178, 2005.

[8] E. Akalin and M. U. Akhmet, "The principles of B-smooth discontinuous flows," Computers $\mathcal{E}$ Mathematics with Applications, vol. 49, no. 7-8, pp. 981-995, 2005.

[9] M. U. Akhmetov and N. A. Perestyuk, "The comparison method for differential equations with impulse action," Differential Equations, vol. 26, no. 9, pp. 1079-1086, 1990.

[10] Y. M. Dib, M. R. Maroun, and Y. N. Raffoul, "Periodicity and stability in neutral nonlinear differential equations with functional delay," Electronic Journal of Differential Equations, vol. 142, pp. 1-11, 2005.

[11] J. K. Hale and S. M. Verduyn Lunel, Introduction to Functional-Differential Equations, vol. 99, Springer, New York, NY, USA, 1993.

[12] K. Gopalsamy, X. Z. He, and L. Z. Wen, "On a periodic neutral logistic equation," Glasgow Mathematical Journal, vol. 33, no. 3, pp. 281-286, 1991.

[13] K. Gopalsamy and B. G. Zhang, "On a neutral delay logistic equation," Dynamics and Stability of Systems, vol. 2, no. 3-4, pp. 183-195, 1987.

[14] I. Győri and F. Hartung, "Preservation of stability in a linear neutral differential equation under delay perturbations," Dynamic Systems and Applications, vol. 10, no. 2, pp. 225-242, 2001.

[15] Y. Li, "Positive periodic solutions of periodic neutral Lotka-Volterra system with state dependent delays," Journal of Mathematical Analysis and Applications, vol. 330, no. 2, pp. 1347-1362, 2007.

[16] M. N. Islam and Y. N. Raffoul, "Periodic solutions of neutral nonlinear system of differential equations with functional delay," Journal of Mathematical Analysis and Applications, vol. 331, no. 2, pp. 1175-1186, 2007.

[17] Y. N. Raffoul, "Periodic solutions for neutral nonlinear differential equations with functional delay," Electronic Journal of Differential Equations, vol. 102, pp. 1-7, 2003.

[18] Y. N. Raffoul, "Periodic solutions for scalar and vector nonlinear difference equations," Panamerican Mathematical Journal, vol. 9, no. 1, pp. 97-111, 1999. 
[19] T. R. Ding, R. Iannacci, and F. Zanolin, “On periodic solutions of sublinear Duffing equations," Journal of Mathematical Analysis and Applications, vol. 158, no. 2, pp. 316-332, 1991.

[20] C. Wang, Y. Li, and Y. Fei, "Three positive periodic solutions to nonlinear neutral functional differential equations with impulses and parameters on time scales," Mathematical and Computer Modelling, vol. 52, no. 9-10, pp. 1451-1462, 2010.

[21] R. W. Leggett and L. R. Williams, "Multiple positive fixed points of nonlinear operators on ordered Banach spaces," Indiana University Mathematics Journal, vol. 28, no. 4, pp. 673-688, 1979. 


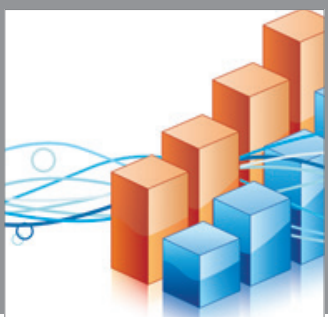

Advances in

Operations Research

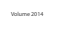

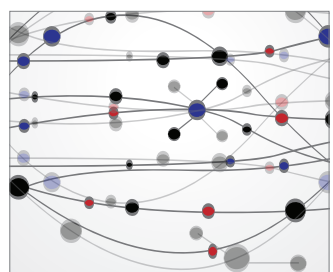

\section{The Scientific} World Journal
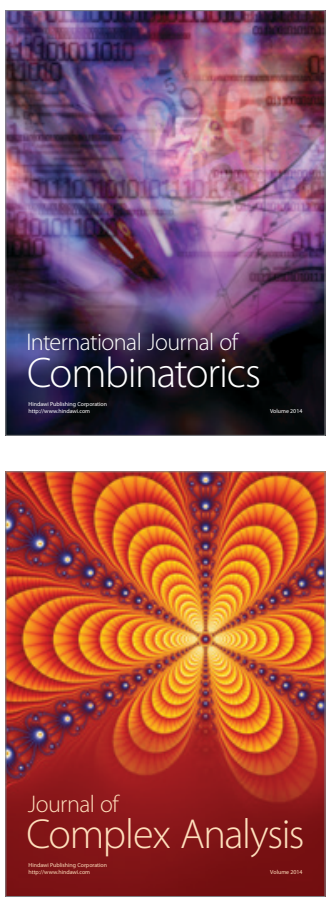

International Journal of

Mathematics and

Mathematical

Sciences
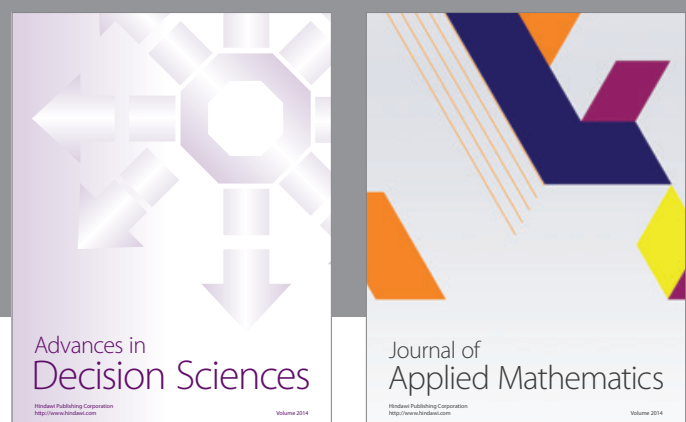

Journal of

Applied Mathematics
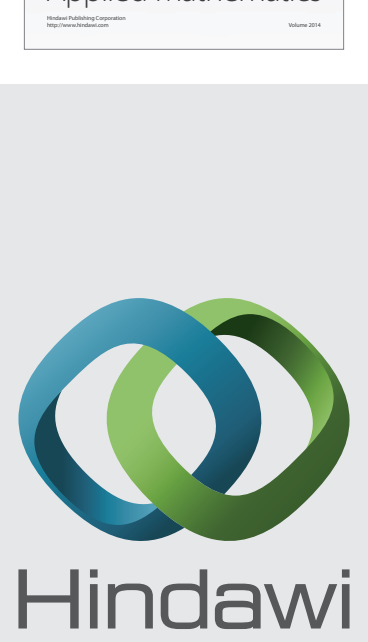

Submit your manuscripts at http://www.hindawi.com
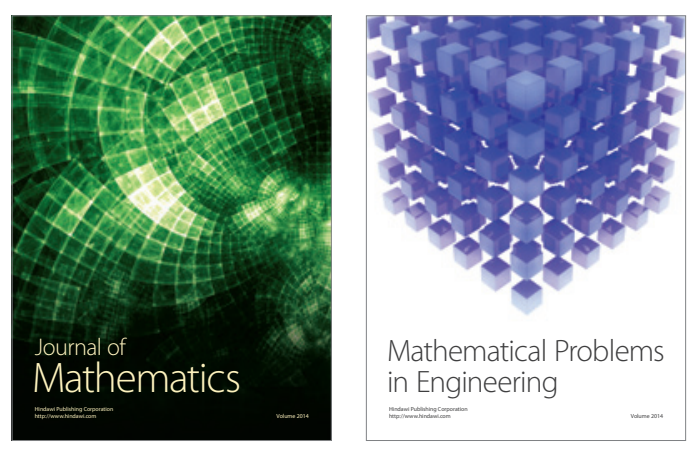

Mathematical Problems in Engineering
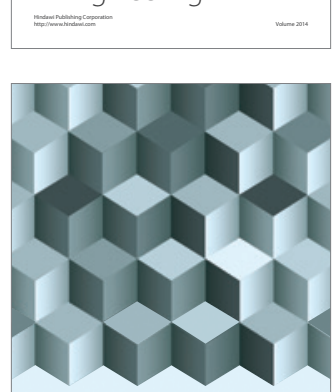

Journal of

Function Spaces
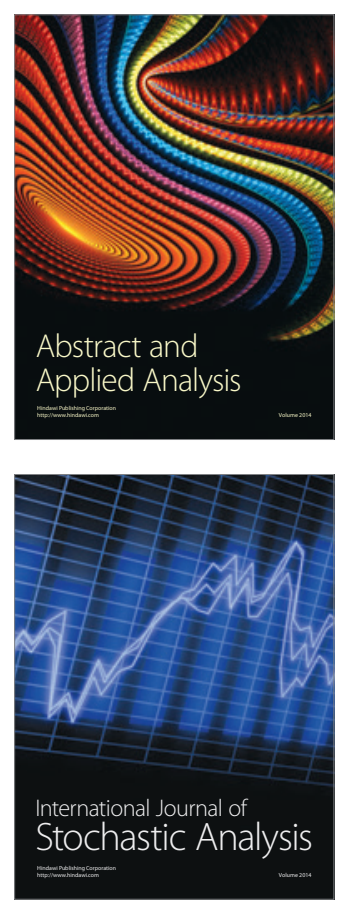

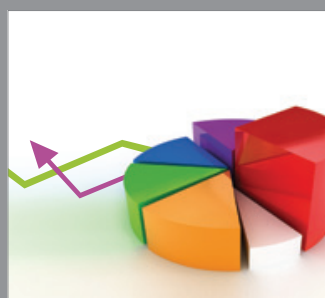

ournal of

Probability and Statistics

Promensencen
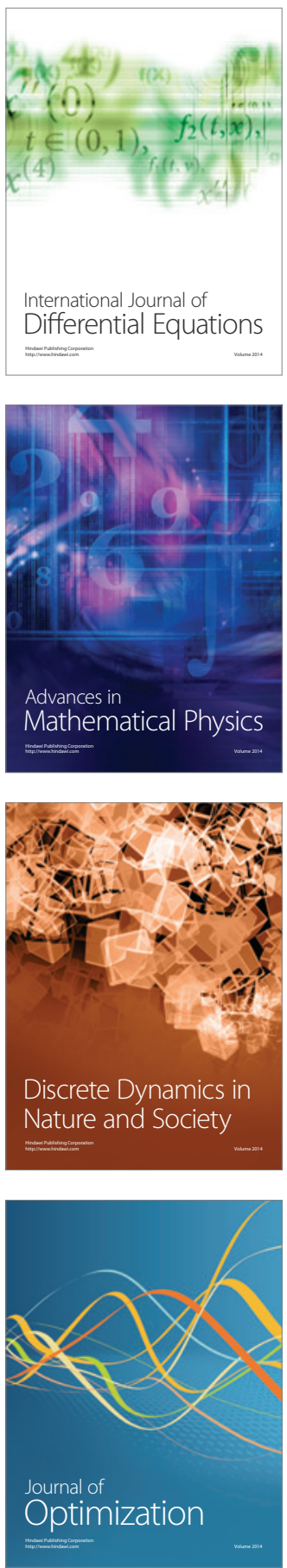